TRANSACTIONS OF THE

AMERICAN MATHEMATICAL SOCIETY

Volume 361, Number 9, September 2009, Pages 4751-4781

S 0002-9947(09)04696-0

Article electronically published on April 21, 2009

\title{
SECOND ORDER CUMULANTS OF PRODUCTS
}

\author{
JAMES A. MINGO, ROLAND SPEICHER, AND EDWARD TAN
}

\begin{abstract}
We derive a formula which expresses a second order cumulant whose entries are products as a sum of cumulants where the entries are single factors. This extends to the second order case the formula of Krawczyk and Speicher. We apply our result to the problem of calculating the second order cumulants of a semi-circular and Haar unitary operator.
\end{abstract}

\section{INTRODUCTION}

In a recent series of papers we have developed the notion of second order freeness [6], [18, 20], 21], 22], 24]. This was motivated by the need for a framework for recent work on the global fluctuations of the eigenvalues of ensembles of random matrices; see e.g. Ambjørn, Jurkiewicz, and Makeenko [1, Anderson and Zeitouni 2, Bai and Silverstein [3, Brézin and Zee [5], Diaconis [8], Johansson [12, Khorunzhy, Khoruzhenko, and Pastur [14.

Free independence, or what we shall call first order freeness, was created by Voiculescu [28, 29] as an adaptation of the usual notion of independence to the noncommutative algebra of matrix valued (or more generally operator valued) random variables. The central object of Voiculescu's theory is called the $R$-transform. For a random variable $a, R(z)$ is a formal power series, which in most examples is an analytic function. The coefficients of $R(z)$ are called the free cumulants of $a$.

The salient feature of Voiculescu's theory is that given the moments of freely independent random variables, there is a universal rule for calculating the moments of sums and products of these random variables. Second order freeness achieves for the fluctuation moments what first order freeness does for ordinary moments. Moreover for many of the standard ensembles of random matrices, independent matrices are asymptotically free of second order, so the theory is quite widely applicable.

Speicher 25] developed a combinatorial approach to free cumulants based on the non-crossing partitions of Kreweras [17. This enabled the theory to be used in many cases where analytic expressions could not be found. In particular in [16], Krawczyk and Speicher found the free analogue of the formula of Leonov and

Received by the editors August 17, 2007.

2000 Mathematics Subject Classification. Primary 46L54; Secondary 15A52, 60F05.

The research of the first and second authors was supported by Discovery Grants and a Leadership Support Initiative Award from the Natural Sciences and Engineering Research Council of Canada.

The research of the second author was supported by a Killam Fellowship from the Canada Council for the Arts.

The research of the third author was supported by an Undergraduate Student Research Award from the Natural Sciences and Engineering Research Council of Canada. 
Shiryaev [19] for calculating cumulants where the entries are products. In this paper we shall extend this result to the second order cumulants introduced in [6].

We begin by motivating the definition of second order cumulants. Let $\left(A_{N}\right)_{N \in \mathbb{N}}$ be a unitarily invariant ensemble of random matrices. By definition this means that the joint distribution of the entries of $A_{N}$ is the same as the joint distribution of the entries of $U A_{N} U^{*}$, where $U$ is an $N \times N$ unitary matrix. We shall say that the ensemble $\left(A_{N}\right)_{N \in \mathbb{N}}$ has a second order limit distribution (cf. [21, 3.4]) if for all integers $p$ and $q$ the limit

$$
\alpha_{p}:=\lim _{N} \frac{1}{N} E\left(\operatorname{Tr}\left(A_{N}^{p}\right)\right)
$$

exists, and for $Y_{N, p}=\operatorname{Tr}\left(A_{N}^{p}-\alpha_{p} I_{N}\right)$ the limit

$$
\alpha_{p, q}:=\lim _{N} E\left(Y_{N, p} Y_{N, q}\right)
$$

exists, and for all $r>2$ and $p_{1}, p_{2}, \ldots, p_{r}$

$$
\lim _{N} k_{r}\left(\operatorname{Tr}\left(A_{N}^{p_{1}}\right), \ldots, \operatorname{Tr}\left(A_{N}^{p_{r}}\right)\right)=0
$$

where $k_{r}$ is the $r^{t h}$ classical cumulant, or semi-invariant (see e.g. 19]). Elements of the sequence $\left(\alpha_{p}\right)_{p}$ are the moments of the limiting distribution. We shall call the elements of the sequence $\left(\alpha_{p, q}\right)_{p, q}$ the fluctuation moments of the limiting distribution.

These two sequences of moments may then be used to define a second order non-commutative probability space on $\mathcal{A}=\mathbb{C}[x]$, the polynomials in the variable $x$. By a second order non-commutative probability space we mean a triple $\left(\mathcal{A}, \phi, \phi_{2}\right)$ where $\mathcal{A}$ is a unital algebra over $\mathbb{C}, \phi: \mathcal{A} \rightarrow \mathbb{C}$ is a tracial linear functional with $\phi(1)=1$, and $\phi_{2}: \mathcal{A} \times \mathcal{A} \rightarrow \mathbb{C}$ is a symmetric bilinear function which is tracial in each variable and $\phi_{2}(1, a)=\phi_{2}(a, 1)=0$ for all $a \in \mathcal{A}$. Thus in our example we let $\phi\left(x^{p}\right)=\alpha_{p}$ and $\phi_{2}\left(x^{p}, x^{q}\right)=\alpha_{p, q}$.

In [6] we introduced the second order $R$-transform, which is a formal power series in two variables: $R(z, w)=\sum_{p, q \geq 1} \kappa_{p, q} z^{p-1} w^{q-1}$. The coefficients $\left(\kappa_{p, q}\right)_{p, q}$ are called the second order cumulants of the second order distribution, and they depend on the first and second order moments $\left(\alpha_{p}\right)_{p}$ and $\left(\alpha_{p, q}\right)_{p, q}$ according to the functional equation given in [6, p. 11].

$$
G(z, w)=G^{\prime}(z) G^{\prime}(w) R(G(z), G(w))+\frac{\partial^{2}}{\partial z \partial w} \log \left(\frac{G(z)-G(w)}{z-w}\right),
$$

where

$$
G(z)=\frac{1}{z} \sum_{p \geq 0} \alpha_{p} z^{-p} \text { and } G(z, w)=\frac{1}{z w} \sum_{p, q \geq 1} \alpha_{p, q} z^{-p} w^{-q} .
$$

Equation (1) determines a sequence of equations relating the moments and the cumulants known as the moment-cumulant relation. Below is a table giving the 
first few equations:

$$
\begin{gathered}
\alpha_{1,1}=\kappa_{1,1}+\kappa_{2}, \\
\alpha_{2,1}=\kappa_{1,2}+2 \kappa_{1} \kappa_{1,1}+2 \kappa_{3}+2 \kappa_{1} \kappa_{2}, \\
\alpha_{2,2}=\kappa_{2,2}+4 \kappa_{1} \kappa_{2,1}+4 \kappa_{1}^{2} \kappa_{1,1}+4 \kappa_{4}+8 \kappa_{1} \kappa_{3}+2 \kappa_{2}^{2}+4 \kappa_{1}^{2} \kappa_{2}, \\
\alpha_{1,3}=\kappa_{1,3}+3 \kappa_{1} \kappa_{2,1}+3 \kappa_{2} \kappa_{1,1}+3 \kappa_{1}^{2} \kappa_{1,1}+3 \kappa_{4}+6 \kappa_{1} \kappa_{3}+3 \kappa_{2}^{2}+3 \kappa_{1}^{2} \kappa_{2}, \\
\alpha_{2,3}=\kappa_{2,3}+2 \kappa_{1} \kappa_{1,3}+3 \kappa_{1} \kappa_{2,2}+3 \kappa_{2} \kappa_{1,2}+9 \kappa_{1}^{2} \kappa_{1,2}+6 \kappa_{1} \kappa_{2} \kappa_{1,1}+6 \kappa_{1}^{3} \kappa_{1,1} \\
+6 \kappa_{5}+18 \kappa_{1} \kappa_{4}+12 \kappa_{2} \kappa_{3}+18 \kappa_{1}^{2} \kappa_{3}+12 \kappa_{1} \kappa_{2}^{2}+6 \kappa_{1}^{3} \kappa_{2}, \\
\alpha_{3,3}=\kappa_{3,3}+6 \kappa_{1} \kappa_{2,3}+6 \kappa_{2} \kappa_{1,3}+6 \kappa_{1}^{2} \kappa_{1,3}+9 \kappa_{1}^{2} \kappa_{2,2}+18 \kappa_{1} \kappa_{2} \kappa_{1,2}+18 \kappa_{1}^{3} \kappa_{1,2} \\
+9 \kappa_{2}^{2} \kappa_{1,1}+18 \kappa_{1}^{2} \kappa_{2} \kappa_{1,1}+9 \kappa_{1}^{4} \kappa_{1,1}+9 \kappa_{6}+36 \kappa_{1} \kappa_{5}+27 \kappa_{2} \kappa_{4}+54 \kappa_{1}^{2} \kappa_{4} \\
+9 \kappa_{3}^{2}+72 \kappa_{1} \kappa_{2} \kappa_{3}+36 \kappa_{1}^{3} \kappa_{3}+12 \kappa_{2}^{3}+36 \kappa_{1}^{2} \kappa_{2}^{2}+9 \kappa_{1}^{4} \kappa_{2} .
\end{gathered}
$$

We shall find it more convenient to use the combinatorial moment cumulant relation for second order cumulants given in [6, Definition 7.4].

Let us recall the combinatorial definition of free cumulants from [23, Lecture 11]. Suppose we have a sequence of multilinear functionals $\left(f_{n}\right)_{n}$ with $f_{n}: \mathcal{A} \times$ $\cdots \times \mathcal{A} \rightarrow \mathbb{C}$ being $n$-linear. We extend this sequence to a family indexed by $\mathcal{P}(n)$, the partitions of $[n]=\{1,2,3, \ldots, n\}$, as follows. If $V=\left\{i_{1}, \ldots, i_{k}\right\} \subset[n]$ we let $f_{V}\left(a_{1}, \ldots, a_{n}\right)=f_{k}\left(a_{i_{1}}, \ldots, a_{i_{k}}\right)$, and if $\pi=\left\{V_{1}, \ldots, V_{t}\right\} \in \mathcal{P}(n)$ we define

$$
f_{\pi}\left(a_{1}, \ldots, a_{n}\right)=f_{V_{1}}\left(a_{1}, \ldots, a_{n}\right) \cdots f_{V_{t}}\left(a_{1}, \ldots, a_{n}\right) .
$$

We can now use this notation to define the free cumulants of a family of random variables $\left\{a_{1}, a_{2}, a_{3}, \ldots\right\} \subset(\mathcal{A}, \phi)$. Let $N C(n) \subset \mathcal{P}(n)$ be the subset of those partitions which are non-crossing [23, Lecture 9]. Then we define multilinear functionals $\left(\kappa_{r}\right)_{r}$ implicitly by the system of equations

$$
\phi\left(a_{1} a_{2} \cdots a_{n}\right)=\sum_{\pi \in N C(n)} \kappa_{\pi}\left(a_{1}, a_{2}, \ldots, a_{n}\right) .
$$

Note that for each $n$ this defines $\kappa_{n}\left(a_{1}, a_{2}, \ldots, a_{n}\right)$ in terms of $\kappa_{p}\left(a_{i_{1}}, \ldots, a_{i_{p}}\right)$ for $p<n$ because $\kappa_{n}$ only occurs once in equation (3), when $\pi=1_{n}=\{1,2, \ldots, n\}$.

The theorem of Krawczyk and Speicher that we extend can now be stated. Let $(\mathcal{A}, \phi)$ be a non-commutative probability space and $a_{1}, \ldots, a_{n_{1}}, a_{n_{1}+1}, \ldots, a_{n_{1}+n_{2}}$, $\ldots, a_{n_{1}+\cdots+n_{p-1}+1}, \ldots, a_{n_{1}+\cdots+n_{p}}$ be elements of $\mathcal{A}$. Let $A_{1}=a_{1} \cdots a_{n_{1}}, A_{2}=$ $a_{n_{1}+1} \cdots a_{n_{1}+n_{2}}, \ldots, A_{p}=a_{n_{1}+\cdots+n_{p-1}+1} \cdots a_{n_{1}+\cdots+n_{p}}$. The problem is to compute $\kappa_{p}\left(A_{1}, A_{2}, \ldots, A_{p}\right)$ in terms of the cumulants $\kappa_{\sigma}\left(a_{1}, \ldots, a_{n}\right)$, where $n=n_{1}+$ $n_{2}+\cdots+n_{p}$. In [16, Theorem 2.2] it is shown that

$$
\kappa_{p}\left(A_{1}, \ldots, A_{p}\right)=\sum_{\sigma \in N C(n)} \kappa_{\sigma}\left(a_{1}, \ldots, a_{n}\right),
$$

where the sum is over all non-crossing partitions $\sigma$ such that $\sigma \vee \tau_{\vec{n}}=1_{n}$ and $\tau_{\vec{n}}=\left\{\left(1, \ldots, n_{1}\right),\left(n_{1}+1, \ldots, n_{1}+n_{2}\right), \ldots,\left(n_{1}+\cdots+n_{p-1}+1, \ldots, n_{1}+\cdots+n_{p}\right)\right\}$ and $1_{n}=\{(1, \ldots, n)\}$ (see Figure 1 ).

The main result of this paper is to prove the analogous result for second order cumulants, viz. to write $\kappa_{p, q}\left(A_{1}, \ldots, A_{p+q}\right)$ in terms of first and second order cumulants of $\left(a_{1}, \ldots, a_{n}\right)$.

To describe the second order cumulants of a second order probability space $\left(\mathcal{A}, \phi, \phi_{2}\right)$ we need the second order equivalent of $N C(n)$. The two parts to this 


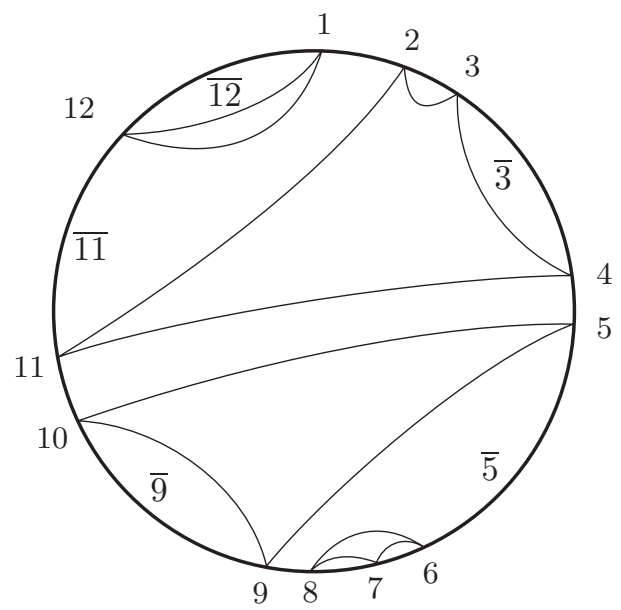

FiguRe 1. In this example $n_{1}=3, n_{2}=2, n_{3}=4, n_{5}=2$, and $n_{6}=1 . \kappa_{5}\left(a_{1} a_{2} a_{3}, a_{4} a_{5}, a_{6} a_{7} a_{8} a_{9}, a_{10} a_{11}, a_{12}\right)$ will be the sum of $\kappa_{\sigma}\left(a_{1}, \ldots, a_{12}\right)$ 's where $\sigma$ runs over all $\sigma$ in $N C(12)$ such that $\sigma \vee \tau_{\vec{n}}=1_{12}$, where $\tau_{\vec{n}}=\{(1,2,3),(4,5),(6,7,8,9),(10,11),(12)\}$. This condition is equivalent to the requirement that the blocks of $\sigma$ separate the points of $\{\overline{3}, \overline{5}, \overline{9}, \overline{11}, \overline{12}\}$.

extension are $(i)$ the notion of a non-crossing annular permutation (see [20, §3] and [22, §2.2]) and (ii) the notion of a non-crossing partitioned permutation (see [6, $\S 4])$.

The non-crossing annular permutations, $S_{N C}(p, q)$, were defined in 20] to be permutations $\pi$ in $S_{p+q}$, the symmetric group of $[p+q]$, which satisfy a geodesic condition:

$$
\#(\pi)+\#\left(\pi^{-1} \gamma_{p, q}\right)+\#\left(\gamma_{p, q}\right)=p+q+2,
$$

where $\#(\pi)$ denotes the number of cycles of $\pi$ and $\gamma_{p, q}$ is the permutation with the two cycles $(1,2,3, \ldots, p)(p+1, \ldots, p+q)$. The cycles of these permutations can be drawn as non-crossing blocks of a $(p, q)$-annulus (see Figure 2 ).

The second notion that we need is that of a non-crossing partitioned permutation. The theory is developed in some generality in [6, §4], but we shall only need a particular case. Let $\pi$ be a permutation in $S_{n}$ and $\mathcal{V}=\left\{V_{1}, V_{2}, \ldots, V_{t}\right\}$ be a partition of $[n]$. If each cycle of $\pi$ is contained in some block of $\mathcal{V}$, then we say that $(\mathcal{V}, \pi)$ is a partitioned permutation. We will frequently write this informally as $\pi \leq \mathcal{V}$.

Definition 1. Let $p$ and $q$ be positive integers. Given $\pi \in S_{N C}(p, q)$ we let $0_{\pi}$ be the partition of $[p+q]$, where the blocks of $\mathcal{V}$ are exactly the cycles of $\pi$. In this way we regard $\pi$ as the partitioned permutation $\left(0_{\pi}, \pi\right)$. Given $\pi=$ $\pi_{1} \times \pi_{2} \in N C(p) \times N C(q)$ and a partition $\mathcal{V}$ of $[p+q]$ such that $\pi \leq \mathcal{V}$ we shall say $(\mathcal{V}, \pi) \in \mathcal{P} \mathcal{S}_{N C}(p, q)^{\prime}$ if all blocks, except one, of $\mathcal{V}$ contain only one cycle of $\pi$, and this exceptional block contains two cycles of $\pi$ - one of $\pi_{1}$ and one of $\pi_{2}$. (See Figure 3.) We let $\mathcal{P} \mathcal{S}_{N C}(p, q)=S_{N C}(p, q) \cup \mathcal{P} \mathcal{S}_{N C}(p, q)^{\prime}$ (cf. [6, Definition 5.13]). 

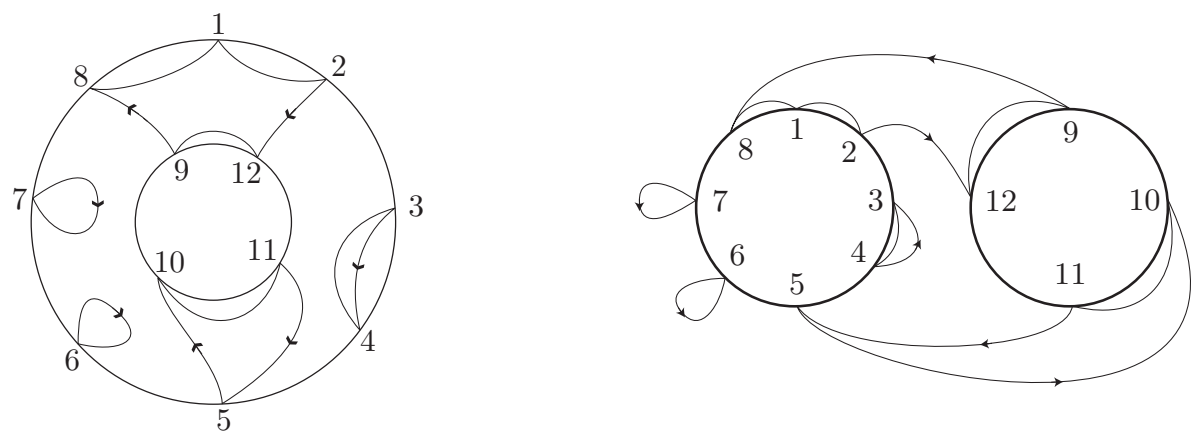

Figure 2. A non-crossing permutation of the $(8,4)$-annulus with cycles $(1,2,12,9,8)(3,4)(5,10,11)(6)(7)$. The same permutation is redrawn with the circles side-by-side.

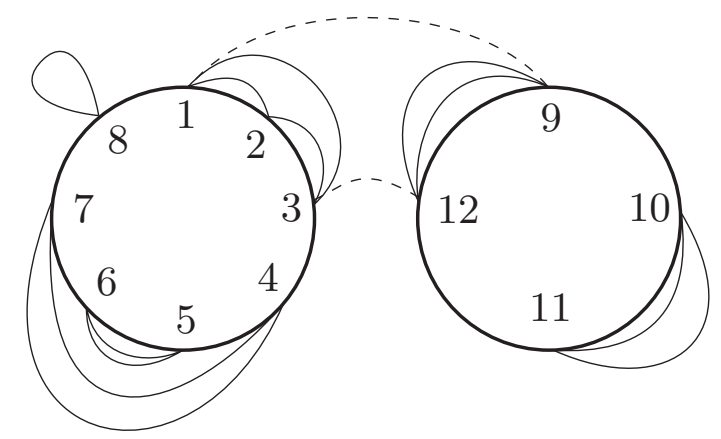

FiguRe 3. A non-crossing partitioned permutation $(\mathcal{V}, \pi)$, where $\pi=(1,2,3)(4,7)(5,6)(8)(9,12)(11,12)$ and $\mathcal{V}=\left\{V_{1}, V_{2}, V_{3}, V_{4}, V_{5}\right\}$ with $V_{1}=(1,2,3,9,12), V_{2}=(4,7), V_{3}=(5,6), V_{4}=(8)$, and $V_{5}=(10,11)$. The block $V_{1}$ contains two cycles of $\pi$ - this is indicated by the dotted line in the diagram; all other blocks of $\mathcal{V}$ contain only one cycle of $\pi$.

Next we wish to extend the definition in equation (2) to the second order case. Suppose we have two sequences $\left(f_{n}\right)_{n}$ and $\left(f_{p, q}\right)_{p, q}$ of multi-linear functionals on a vector space with $f_{r}$ being $r$-linear and invariant under cyclic permutation of its arguments, and $f_{p, q}$ being $p+q$-linear and invariant under a cyclic permutation of the first $p$ arguments or the last $q$ arguments. Let $(\mathcal{V}, \pi)$ be a partitioned permutation where each block of $\mathcal{V}$ contains either one or two cycles of $\pi$. If $V$ is a block of $\mathcal{V}$ containing only one cycle $\left(i_{1}, \ldots, i_{s}\right)$ of $\pi$, then we define $f_{V}\left(a_{1}, \ldots, a_{p+q}\right)$ to be $f_{s}\left(a_{i_{1}}, \ldots, a_{i_{s}}\right)$, as was done for equation (11). If $V$ contains two cycles $\left(i_{1}, \ldots, i_{s}\right)$ and $\left(j_{1}, \ldots, j_{t}\right)$ of $\pi$, then we define $f_{V}\left(a_{1}, \ldots, a_{p+q}\right)$ to be $f_{s, t}\left(a_{i_{1}}, \ldots, a_{i_{s}}, a_{j_{1}}, \ldots, a_{j_{t}}\right)$. We then define

$$
f_{(\mathcal{V}, \pi)}\left(a_{1}, \ldots, a_{p+q}\right)=f_{V_{1}}\left(a_{1}, \ldots, a_{p+q}\right) \cdots f_{V_{l}}\left(a_{1}, \ldots, a_{p+q}\right),
$$

where $\mathcal{V}=\left\{V_{1}, V_{2}, \ldots, V_{l}\right\}$.

Definition 2. Let $\left(\mathcal{A}, \phi, \phi_{2}\right)$ be a second order probability space and $\left(\kappa_{n}\right)_{n}$ the first order cumulants given by equation (3). We define recursively second order 
cumulants $\left(\kappa_{p, q}\right)_{p, q}$ which will be multi-linear functionals on $\mathcal{A}$ by the system of equations

$$
\phi_{2}\left(a_{1} \cdots a_{p}, a_{p+1} \cdots a_{p+q}\right)=\sum_{(\mathcal{V}, \pi) \in \mathcal{P} \mathcal{S}_{N C}(p, q)} \kappa_{(\mathcal{V}, \pi)}\left(a_{1}, \ldots, a_{p+q}\right) .
$$

In equation (77) the term $\kappa_{p, q}\left(a_{1}, \ldots, a_{p+q}\right)$ only occurs once: when $\mathcal{V}=1_{p+q}$ and $\pi=\gamma_{p, q}$, where $\gamma_{p, q}$ is the permutation with two cycles $(1,2, \ldots, p)(p+1, \ldots, p+q)$. For all other $(\mathcal{V}, \pi), \kappa_{(\mathcal{V}, \pi)}$ is a product of $\kappa_{n}$ 's and $\kappa_{r, s}$ 's with either $r<p$ or $s<q$.

It is usually convenient to write equation (7) as a sum with two terms: the first term only involves first order cumulants and the second term involves both orders of cumulants. Recall that $\mathcal{P} \mathcal{S}_{N C}(p, q)=S_{N C}(p, q) \cup \mathcal{P} \mathcal{S}_{N C}(p, q)^{\prime}$, and so

$$
\begin{aligned}
\phi_{2}\left(a_{1} \cdots a_{p}, a_{p+1} \cdots a_{p+q}\right)= & \sum_{\pi \in S_{N C}(p, q)} \kappa_{\pi}\left(a_{1}, \ldots, a_{p+q}\right) \\
& +\sum_{(\mathcal{V}, \pi) \in \mathcal{P} \mathcal{S}_{N C}(p, q)^{\prime}} \kappa_{(\mathcal{V}, \pi)}\left(a_{1}, \ldots, a_{p+q}\right) .
\end{aligned}
$$

To illustrate this definition let us work out the first few second order cumulants. When $p=q=1$ equation (6) becomes

$$
\phi_{2}(a, b)=\kappa_{2}(a, b)+\kappa_{1,1}(a, b)
$$

because there are two elements in $\mathcal{P} \mathcal{S}_{N C}(1,1)$; the first is when $(\mathcal{V}, \pi)=\left(1_{2},(1,2)\right)$ and the second when $(\mathcal{V}, \pi)=\left(1_{2},(1)(2)\right)$. Here we are writing the permutations in cycle notation and $1_{2}$ is the partition of [2] that has one block. By equation (3) $\kappa_{2}(a, b)=\phi(a b)-\phi(a) \phi(b)$. So solving for $\kappa_{1,1}(a, b)$ we obtain that

$$
\kappa_{1,1}(a, b)=\phi_{2}(a, b)+\phi(a) \phi(b)-\phi(a b) .
$$

Let us next look at the case $p=2$ and $q=1$. There are four partitioned permutations $(\mathcal{V}, \pi)$ with $\mathcal{V}=0_{\pi}$. They are $\pi_{1}=(1,3,2), \pi_{2}=(1,2,3), \pi_{3}=$ $(1,3)(2)$ and $\pi_{4}=(1)(2,3)$. These are the four non-crossing annular permutations of a $(2,1)$-annulus (see Figure 4$)$. The contribution of these four diagrams is $\kappa_{3}\left(a_{1}, b, a_{2}\right)+\kappa_{3}\left(a_{1}, a_{2}, b\right)+\kappa_{2}\left(a_{1}, b\right) \kappa_{1}\left(a_{2}\right)+\kappa_{1}\left(a_{1}\right) \kappa_{2}\left(a_{2}, b\right)$.

There are three diagrams that involve second order cumulants; they correspond to the three partitioned permutations, $\left(1_{3}, \gamma_{2,1}\right),\left(\mathcal{V}_{1}, e\right)$, and $\left(\mathcal{V}_{2}, e\right)$, where $1_{3}=((1,2,3)), \gamma_{2,1}=(1,2)(3), e=(1)(2)(3)$ is the identity permutation, $\mathcal{V}_{1}=$ $\{(1,3),(2)\}$, and $\mathcal{V}_{2}=\{(1),(2,3)\}$ (see Figure 5 ).

The contribution of these three diagrams is $\kappa_{2,1}\left(a_{1}, a_{2}, b\right)+\kappa_{1,1}\left(a_{1}, b\right) \kappa_{1}\left(a_{2}\right)+$ $\kappa_{1}\left(a_{1}\right) \kappa_{1,1}\left(a_{2}, b\right)$. Note that second order cumulants only appear when a block of $\mathcal{V}$ connects two cycles of $\pi$. Putting together all the terms we see that

$$
\begin{aligned}
\kappa_{2,1}\left(a_{1}, a_{2}, b\right)= & \phi_{2}\left(a_{1} a_{2}, b\right)-\phi\left(a_{1}\right) \phi_{2}\left(a_{2}, b\right)-\phi\left(a_{2}\right) \phi_{2}\left(a_{1}, b\right) \\
& -\phi\left(a_{1} a_{2} b\right)-\phi\left(a_{1} b a_{2}\right)+2 \phi\left(a_{1}\right) \phi\left(a_{2} b\right) \\
& +2 \phi\left(a_{1} b\right) \phi\left(a_{2}\right)+2 \phi\left(a_{1} a_{2}\right) \phi(b) \\
& -4 \phi\left(a_{1}\right) \phi\left(a_{2}\right) \phi(b) .
\end{aligned}
$$

In [6, Definition 7.4] a general formula was given for writing any cumulant in terms of the moments using the higher order Möbius function. We shall prefer to use equation (77).

In equation (4) the condition on $\pi$ was that $\pi \vee \tau_{\vec{r}}=1_{n}$. We shall show that this is equivalent to the condition that $\pi^{-1} \gamma_{n}$ separates the points of $N=\left\{n_{1}, n_{1}+\right.$ 

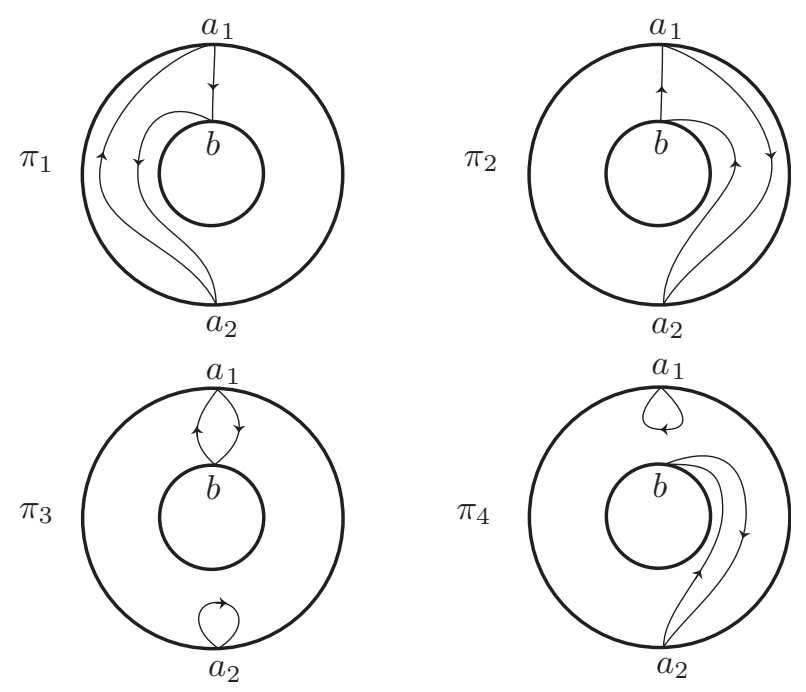

Figure 4. The four non-crossing permutations of a $(2,1)$-annulus.
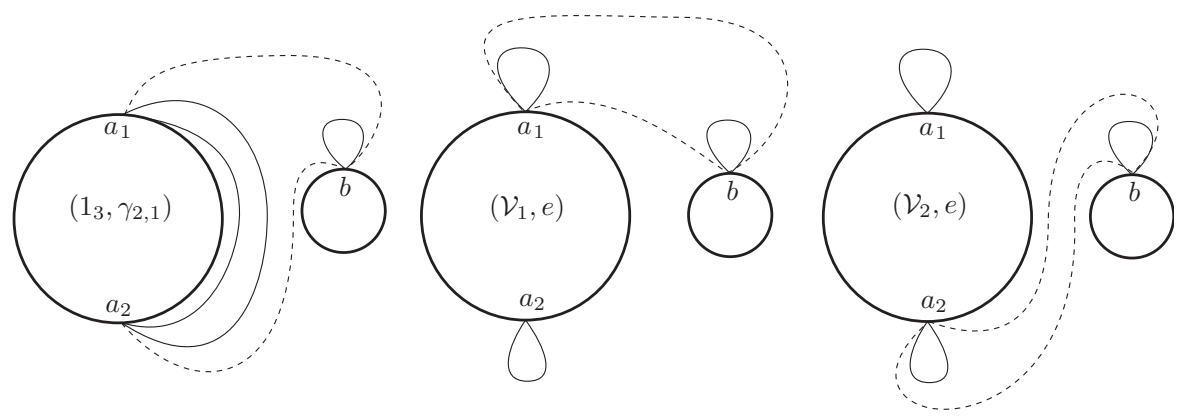

FiguRE 5. The three diagrams that involve second order cumulants.

$\left.n_{2}, \ldots, n_{1}+\cdots+n_{r}\right\}$, i.e. that no two points of $N$ lie in the same cycle of $\pi^{-1} \gamma_{n}$. When we pass to the annular case this condition becomes that $\pi^{-1} \gamma_{p, q}$ separates the points of $N$.

Theorem 3. Suppose $n_{1}, \ldots, n_{r}, n_{r+1}, \ldots, n_{r+s}$ are positive integers, $p=n_{1}+$ $\cdots+n_{r}, q=n_{r+1}+\cdots+n_{r+s}$, and

$$
N=\left\{n_{1}, n_{1}+n_{2}, \ldots, n_{1}+\cdots+n_{r+s}\right\} .
$$

Given a second order probability space $\left(\mathcal{A}, \phi, \phi_{2}\right)$ and

$$
a_{1}, \ldots, a_{n_{1}}, a_{n_{1}+1}, \ldots, a_{n_{1}+n_{2}}, \ldots, a_{n_{1}+\cdots+n_{r+s}} \in \mathcal{A},
$$

let $A_{1}=a_{1} \cdots a_{n_{1}}, \ldots, A_{r+s}=a_{n_{1}+\cdots+n_{r+s-1}+1} \cdots a_{n_{1}+\cdots+n_{r+s}}$. Then

$$
\kappa_{r, s}\left(A_{1}, \ldots, A_{r}, A_{r+1}, \ldots, A_{r+s}\right)=\sum_{(\mathcal{V}, \pi)} \kappa_{(\mathcal{V}, \pi)}\left(a_{1}, \ldots, a_{p+q}\right),
$$

where the summation is over those $(\mathcal{V}, \pi) \in \mathcal{P} \mathcal{S}_{N C}(p, q)$ such that $\pi^{-1} \gamma_{p, q}$ separates the points of $N$. 
In section 2 we shall present some preliminary results on non-crossing permutations needed for the proof of the main theorem, which will appear in section 3 . In section 4 we will give our first application, namely the computation of the second order cumulants of the square of a semi-circular operator. In section 5, our second application will be to the second order cumulants of a Haar unitary. In section 6 we present a partial order on partitioned permutations that extends in a very natural way the usual order on $N C(n)$. This shows how the preparatory remarks in section 2 can be understood in terms of the extension of Kreweras's complement to partitioned permutations.

\section{Preliminaries ON NON-CROSSING PERMUtATIONS}

In this section we shall recall the notation we shall need for the proof of the main theorem and translate the criterion of equation (4) into a criterion appropriate to the annular case: Proposition 25.

Notation 4. Let $\pi \in S_{n}$ and let $\#(\pi)$ be the number of cycles in the cycle decomposition of $\pi$. Let $\gamma_{n}=(1,2,3, \ldots, n)$. We put the metric on $S_{n}$ given by $|\pi|=d(e, \pi)=n-\#(\pi)$, where $e$ is the identity permutation and $d(\pi, \sigma)=$ $d\left(e, \pi^{-1} \sigma\right)=d\left(e, \sigma \pi^{-1}\right)$. For $\pi \in S_{n}$ to be in $N C(n)$ it is necessary and sufficient that

$$
\#(\pi)+\#\left(\pi^{-1} \gamma_{n}\right)+\#\left(\gamma_{n}\right)=n+2
$$

which is equivalent to the condition in Biane [4]

$$
|\pi|+\left|\pi^{-1} \gamma_{n}\right|=\left|\gamma_{n}\right|
$$

In addition, Biane showed that for $\pi, \sigma \in N C(n)$ the relation $\pi \leq \sigma$ is equivalent to the equation $|\pi|+\left|\pi^{-1} \sigma\right|=|\sigma|$, where the relation $\pi \leq \sigma$ means that each cycle of $\pi$ is contained in some cycle of $\sigma$. Recall that with this partial order $N C(n)$ is a lattice; the supremum of two non-crossing permutations will be denoted $\pi \vee \sigma$.

For $\pi \in S_{p+q}$ to be in $S_{N C}(p, q)$ it is necessary and sufficient (see [20]) that at least one cycle of $\pi$ connects the two cycles of $\gamma_{p, q}$ and

$$
\#(\pi)+\#\left(\pi^{-1} \gamma_{p, q}\right)+\#\left(\gamma_{p, q}\right)=p+q+2
$$

where $\gamma_{p, q}$ is the permutation with two cycles $(1,2,3, \ldots, p)(p+1, \ldots, p+q)$. In terms of the metric this condition becomes $|\pi|+\left|\pi^{-1} \gamma_{p, q}\right|=\left|\gamma_{p, q}\right|+2$. In [6, §4.5] a general theory of length of a partitioned permutation is given.

Let $\pi \in S_{n}$ and let $N \subset[n]$ be a subset. If $\pi$ leaves $N$ invariant we denote by $\left.\pi\right|_{N}$ the restriction of $\pi$ to $N$. If $\pi$ does not leave $N$ invariant we can still define a permutation on $N$ as follows. If $p \in N$ and $\pi(p) \notin N$, then $\left.\pi\right|_{N}(p)=\pi^{k}(p)$, where $k$ is the smallest integer such that $\pi^{k-1}(p) \notin N$ but $\pi^{k}(p) \in N$. Let $N C(N)$ denote the non-crossing permutations of $N$ where the points are in the same order as they are in $[n]$. If $\pi$ is in $N C(n)$, then $\left.\pi\right|_{N}$ is in $N C(N)$, as a crossing for $\left.\pi\right|_{N}$ would also be a crossing for $\pi$.

Let $p$ and $q$ be positive integers and $N \subset[p+q]$. Let $N_{1}=N \cap[p]$ and $N_{2}=N \cap[p+1, p+q]$. We denote by $S_{N C}\left(N_{1}, N_{2}\right)$ the non-crossing annular permutations of $N$ arranged on an annulus with the points of $N_{1}$ on the outer circle and the points of $N_{2}$ on the inner circle. 
Lemma 5. Given $\pi \in S_{N C}(p, q),\left.\pi\right|_{N} \in S_{N C}\left(N_{1}, N_{2}\right) \cup(N C(p) \times N C(q))$.

Proof. By [20, Equation 5.2] there are integers $u$ and $v$ such that $\tilde{\pi}=\gamma_{p}^{u} \gamma_{q}^{v} \pi \gamma_{q}^{-v} \gamma_{p}^{-u}$ $\in N C(p+q)$. Let $\tilde{N}=\gamma_{p}^{u} \gamma_{q}^{v}(N)$. Then $\left.\tilde{\pi}\right|_{\tilde{N}} \in N C(\tilde{N})$, and $\left.\pi\right|_{N}$ and $\left.\tilde{\pi}\right|_{\tilde{N}}$ are conjugate. Thus $\left.\pi\right|_{N} \in S_{N C}\left(N_{1}, N_{2}\right) \cup N C(p) \times N C(q)$.

Lemma 6. Let $N$ be a subset of $[n]$ and $\sigma, \pi \in S_{n}$ such that $\pi(i)=i$ for $i \notin N$ (and thus $\pi$ leaves $N$ invariant). Then $\left.(\sigma \pi)\right|_{N}=\left.\left.\sigma\right|_{N} \pi\right|_{N}$.

Proof. Let $m \in N$ and suppose $\left.(\sigma \pi)\right|_{N}(m)=(\sigma \pi)^{k}(m)$. Then for $1 \leq i<k$, $\sigma^{i}(\pi(m)) \notin N$ and $\sigma^{k}(\pi(m)) \in N$. Thus $\left.\sigma^{i}\right|_{N}(\pi(m)) \notin N$ for $1 \leq i<k$ and $\sigma^{k}(\pi(m)) \in N$. Hence $\left.\sigma\right|_{N}(\pi(m))=\left.(\sigma \pi)\right|_{N}(m)$ and $\left.\left.\sigma\right|_{N} \pi\right|_{N}=\left.(\sigma \pi)\right|_{N}$.

The next lemma is a special case of [6, Proposition 4.10].

Lemma 7. Suppose $\pi, \sigma$, and $\tau$ are in $S_{n}$ and $|\pi|+\left|\pi^{-1} \sigma\right|=|\sigma|$ and $|\sigma|+\left|\sigma^{-1} \tau\right|=$ $|\tau|$. Then $|\pi|+\left|\pi^{-1} \tau\right|=|\tau|$.

In [4, Theorem 1] Biane showed that if $\sigma$ has only one cycle and $|\pi|+\left|\pi^{-1} \sigma\right|=|\sigma|$, then the cycles of $\pi$ form a non-crossing partition of $\sigma$. We shall need the extension of this to the case where $\sigma$ has more than one cycle.

Lemma 8. Suppose $\pi, \sigma \in S_{n}$ and $|\pi|+\left|\pi^{-1} \sigma\right|=|\sigma|$. Then:

(i) each cycle of $\pi$ is contained in some cycle of $\sigma$, and

(ii) for each cycle $c$ of $\sigma$ the enclosed cycles of $\pi$ form a non-crossing partition of $c$.

Proof. First let us show $(i)$. Suppose that $a$ and $b$ are in the same cycle of $\pi$. We must show that they are in the same cycle of $\sigma$. If $a$ and $b$ are in the same cycle of $\pi$, then writing the transposition that switches $a$ and $b$ as $(a, b)$ we have

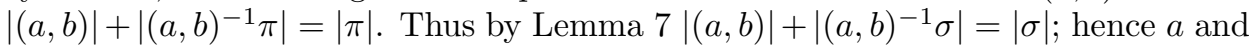
$b$ are in the same cycle of $\sigma$.

Let us show that $(i i)$ then follows from [4, Theorem 1].

Write $\sigma=c_{1} c_{2} \cdots c_{k}$ as a product of cycles and let $\pi_{i}$ be the product of cycles of $\pi$ contained in the cycle $c_{i}$. For each $i$ we have $\left|c_{i}\right| \leq\left|\pi_{i}\right|+\left|\pi_{i}^{-1} c_{i}\right|$. Thus

$$
\begin{aligned}
|\sigma|= & \left|c_{1}\right|+\cdots+\left|c_{k}\right| \leq\left|\pi_{1}\right|+\cdots+\left|\pi_{k}\right| \\
& +\left|\pi_{1}^{-1} c_{1}\right|+\cdots+\left|\pi_{k}^{-1} c_{k}\right|=|\pi|+\left|\pi^{-1} \sigma\right|=|\sigma| .
\end{aligned}
$$

Thus for each $i$ we must have equality in the inequality $\left|c_{i}\right| \leq\left|\pi_{i}\right|+\left|\pi_{i}^{-1} c_{i}\right|$. Hence (ii).

Definition 9. (i) Let $n_{1}, n_{2}, \ldots, n_{r}$ be positive integers and $n=n_{1}+n_{2}+\cdots+n_{r}$. Given a permutation $\pi \in S_{r}$ we shall define a permutation $\pi_{\vec{n}} \in S_{n}$ as follows. Let $N=\left\{n_{1}, n_{1}+n_{2}, \ldots, n_{1}+n_{2}+\cdots+n_{r}\right\}$. For $i \notin N$ let $\pi_{\vec{n}}(i)=i+1$ and $\pi_{\vec{n}}\left(n_{1}+\cdots+n_{k}\right)=n_{1}+\cdots+n_{\pi(k)-1}+1$.

(ii) Let $\tau$ be the partition of $[r]$ in which all blocks are singletons.

This definition may be illustrated as follows. First $\tau_{\vec{n}}=\left\{T_{1}, T_{2}, \ldots, T_{r}\right\}$, where $T_{i}$ is the cycle $\left(n_{1}+\cdots+n_{i-1}+1, n_{1}+\cdots+n_{i-1}+2, \ldots, n_{1}+\cdots+n_{i}\right) \cdot \pi_{\vec{n}}$ takes the last element of $T_{i}$ to the first element of $T_{\pi(i)}$; elements not in $N$ are increased by 1 . If $\mathcal{V}$ is a partition of $[r]$, we let $\mathcal{V}_{\vec{n}}=\mathcal{V} \vee \tau_{\vec{n}}$. 

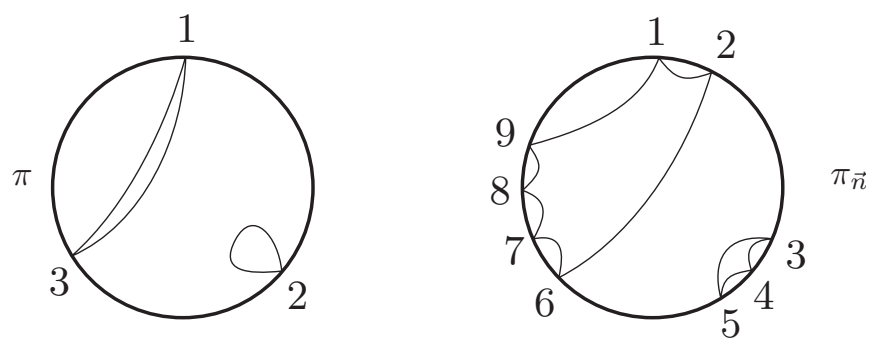

FiguRE 6. $\pi \in S_{3}$ and $\pi_{\vec{n}} \in S_{9}$.

Example 10. Let $n_{1}=2, n_{2}=3, n_{3}=4$ and $\pi=(1,3)(2)$. Then $\pi_{\vec{n}}=$ $(1,2,6,7,8,9)(3,4,5)$ (see Figure 6).

Remark 11. Since $\pi_{\vec{n}}$ takes the last element of $T_{i}$ to the first element of $T_{\pi(i)}, \pi_{\vec{n}}^{-1}$ takes the first element of $T_{i}$ to the last element of $T_{\pi^{-1}(i)}$. Thus

$$
\pi_{\vec{n}}^{-1} \gamma_{n}(i)= \begin{cases}i, & i \notin N, \\ n_{1}+\cdots+n_{\pi^{-1}(j+1)}, & i=n_{1}+\cdots+n_{j} .\end{cases}
$$

This last case can also be written as $\pi_{\vec{n}}^{-1} \gamma_{n}\left(n_{1}+\cdots+n_{j}\right)=n_{1}+\cdots+n_{\pi^{-1} \gamma_{r}(j)}$.

Lemma 12. Let $\psi:[r] \rightarrow[n]$ be defined by $\psi(i)=n_{1}+\cdots+n_{i}$. Then

(i) $\psi \pi^{-1} \gamma_{r}=\pi_{\vec{n}}^{-1} \gamma_{n} \psi$,

(ii) $\pi \in N C(r)$ if and only if $\pi_{\vec{n}} \in N C(n)$.

Proof. Part $(i)$ follows from the previous remark. Therefore $\#(\pi)=\#\left(\pi_{\vec{n}}\right)$ and $\#\left(\pi_{\vec{n}}^{-1} \gamma_{n}\right)=\#\left(\pi^{-1} \gamma_{r}\right)+n-r$, by part $(i)$. Thus

$$
\#\left(\pi_{\vec{n}}\right)+\#\left(\pi_{\vec{n}}^{-1} \gamma_{n}\right)+\#\left(\gamma_{n}\right)=\#(\pi)+\#\left(\pi_{\vec{n}}^{-1} \gamma_{r}\right)+\#\left(\gamma_{r}\right)+n-r .
$$

Hence $\#\left(\pi_{\vec{n}}\right)+\#\left(\pi_{\vec{n}}^{-1} \gamma_{n}\right)+\#\left(\gamma_{n}\right)=n+2$ if and only if $\#(\pi)+\#\left(\pi^{-1} \gamma_{r}\right)+\#\left(\gamma_{r}\right)=$ $r+2$

Lemmas 13 and 19 have an interpretation in terms of the relative Kreweras complement $K_{\sigma}(\pi)=\pi^{-1} \sigma$ (see e.g. Nica-Speicher [23, Def. 18.3]). The permutation $\sigma \pi^{-1}$ could be called the "right hand" Kreweras complement, $\tilde{K}_{\sigma}(\pi)$. Lemma 13 then says that $\tau \leq K_{\gamma}(\sigma) \Leftrightarrow \sigma \leq \tilde{K}_{\gamma}(\tau)$. In Lemma 19 this is extended to the annular case. In section 6 we explain how this analogy with the Kreweras complement can be made precise by extending the order on $N C(n)$ to non-crossing partitioned permutations.

Lemma 13. Let $\tau, \sigma, \gamma \in N C(n)$ with $\tau, \sigma \leq \gamma$. Then $\tau \leq \sigma^{-1} \gamma$ if and only if $\sigma \leq \gamma \tau^{-1}$.

Proof. If $\tau \leq \sigma^{-1} \gamma$, then $|\tau|+\left|\tau^{-1} \sigma^{-1} \gamma\right|=\left|\sigma^{-1} \gamma\right|$. Since $\sigma \leq \gamma$ we also have $|\sigma|+\left|\sigma^{-1} \gamma\right|=|\gamma|$. Thus

$$
\begin{aligned}
& |\sigma|+\left|\sigma^{-1} \gamma \tau^{-1}\right|=|\gamma|-\left|\sigma^{-1} \gamma\right|+\left|\tau^{-1} \sigma^{-1} \gamma\right| \\
& \quad=|\gamma|-\left|\sigma^{-1} \gamma\right|+\left|\sigma^{-1} \gamma\right|-|\tau| \\
& \quad=|\gamma|-|\tau|=\left|\gamma \tau^{-1}\right|
\end{aligned}
$$

and therefore $\sigma \leq \gamma \tau^{-1}$. 


$$
\begin{aligned}
& \text { If } \sigma \leq \gamma \tau^{-1} \text {, then }|\sigma|+\left|\sigma^{-1} \gamma \tau^{-1}\right|=\left|\gamma \tau^{-1}\right| \text {; also }|\tau|+\left|\tau^{-1} \gamma\right|=|\gamma| \text {. Thus } \\
& \qquad \begin{aligned}
&|\tau|+\left|\tau^{-1} \sigma^{-1} \gamma\right|=|\gamma|-\left|\gamma \tau^{-1}\right|+\left|\sigma^{-1} \gamma \tau^{-1}\right| \\
&=|\gamma|-\left|\gamma \tau^{-1}\right|+\left|\gamma \tau^{-1}\right|-|\sigma| \\
&=\left|\sigma^{-1} \gamma\right|=\left|\sigma^{-1} \gamma\right|,
\end{aligned}
\end{aligned}
$$

which shows that $\tau \leq \sigma^{-1} \gamma$.

Lemma 14. For $\sigma \in N C(n), \sigma \vee \tau_{\vec{n}}=\gamma_{n}$ if and only if $\sigma^{-1} \gamma_{n}$ separates the points of $N$.

Proof. We have $\tau_{\vec{n}}^{-1} \gamma_{n}\left(n_{1}+\cdots+n_{k}\right)=n_{1}+\cdots+n_{k+1}$, and thus for $r \neq s \in N$, $(r, s) \leq \tau_{\vec{n}}^{-1} \gamma_{n}$ and by the lemma above, $\tau_{\vec{n}} \leq \gamma_{n}(r, s)$. Hence, if $r \neq s \in N$ are in the same cycle of $\sigma^{-1} \gamma_{n}$, then by the lemma above $\sigma \leq \gamma_{n}(r, s)$ and hence $\sigma \vee \tau_{\vec{n}} \leq \gamma_{n}(r, s)$.

Conversely if $\sigma \vee \tau_{\vec{n}}<\gamma_{n}$, then there must be $r \neq s \in N$ such that $\sigma \vee \tau_{\vec{n}} \leq$ $\gamma_{n}(r, s)$, as partitions of the form $\gamma_{n}(r, s)$ with $r \neq s \in N$ are the most general partitions with two blocks, each of which is a union of $T_{i}$ 's. In this case $(r, s) \leq$ $\sigma^{-1} \gamma_{n}$ and $\sigma^{-1} \gamma_{n}$ fails to separate the points of $N$.

Theorem 15. For $\sigma \in N C(n)$ with $\sigma \leq \pi_{\vec{n}}$ we have that $\sigma \vee \tau_{\vec{n}}=\pi_{\vec{n}}$ if and only if $\sigma^{-1} \pi_{\vec{n}}$ separates the points of $N$.

Proof. Let the cycle decomposition of $\pi$ be $c_{1} c_{2} \cdots c_{k}$. Then the cycle decomposition of $\pi_{\vec{n}}$ is $\tilde{c}_{1} \tilde{c}_{2} \cdots \tilde{c}_{k}$, where $\tilde{c}_{i}=\left(c_{i}\right)_{\vec{n}}$. Let $\sigma_{i}$ be the restriction of $\sigma$ to the invariant subset consisting of the points of the cycle $\tilde{c}_{i}$; similarly let $\tau_{i}$ be the restriction of $\tau_{\vec{n}}$ to the same invariant subset. Thus $\sigma \vee \tau_{\vec{n}}=\pi_{\vec{n}}$ if and only if for each $i$ we have $\sigma_{i} \vee \tau_{i}=\tilde{c}_{i}$, and by the previous lemma we have that $\sigma_{i} \vee \tau_{i}=\tilde{c}_{i}$ for each $i$ if and only if for each $i, \sigma_{i}^{-1} \tilde{c}_{i}$ separates the points of $N \cap \tilde{c}_{i}$. Since for each $i, \pi_{\vec{n}}, \sigma$, and $\tau_{\vec{n}}$ leave $\tilde{c}_{i}$ invariant, we have that $\sigma^{-1} \pi_{\vec{n}}$ separates the points of $N \cap \tilde{c}_{i}$ for each $i$ if and only if $\sigma^{-1} \pi_{\vec{n}}$ separates the points of $N$.

Remark 16. The condition that $\sigma^{-1} \gamma_{n}$ separates the points of $N$ can be rephrased by saying that any two points in the same cycle of $\tau_{\vec{n}}^{-1} \gamma_{n}$ must be in different cycles of $\sigma^{-1} \pi_{\vec{n}}$.

Definition 17. Let $\pi, \sigma \in S_{N C}(p, q)$. Suppose that each cycle of $\pi$ is contained in some cycle of $\sigma$ and for each cycle $c$ of $\sigma$ the enclosed cycles of $\pi$ form a noncrossing partition of $c$. Then we write $\pi \leq \sigma$. By Lemma 8 this relation extends to $S_{N C}(p, q)$ the usual partial order on $N C(n)$ given by inclusion of blocks.

Remark 18. We can rephrase the definition above using our metric $d$. Write the cycles of $\pi$ as $c_{1} c_{2} \cdots c_{l}$ and denote the restriction of $\pi$ to $c_{i}$ by $\pi_{i}$. Then we have

$$
\#\left(\pi_{i}\right)+\#\left(\pi_{i}^{-1} c_{i}\right)+\#\left(c_{i}\right)=k_{i}+2
$$

where $k_{i}$ is the length of the cycle $c_{k}$. If we sum over $i$ we get

$$
\#(\pi)+\#\left(\pi^{-1} \sigma\right)+\#(\sigma)=p+q+2 l,
$$

which can be rewritten $|\pi|+\left|\pi^{-1} \sigma\right|=|\sigma|$ (cf. [6, Definition 4.9]). That the relation $\leq$ is transitive follows from [6, Proposition 4.10], or in this simple case it can be checked directly. 
Moreover, we can extend Definition 17 as follows. Suppose that $\pi \in S_{p+q}, \sigma \in$ $S_{N C}(p, q)$ and each cycle of $\pi$ is contained in a cycle of $\sigma$ and that for each cycle of $\sigma$ the enclosed cycles of $\pi$ form a non-crossing permutation of this cycle of $\sigma$. By [20, Equation 5.2] there are integers $u$ and $v$ such that $\tilde{\sigma}=\gamma_{p}^{u} \gamma_{q}^{v} \sigma \gamma_{q}^{-v} \gamma_{p}^{-u} \in N C(p+q)$. Let $\tilde{\pi}=\gamma_{p}^{u} \gamma_{q}^{v} \pi \gamma_{q}^{-v} \gamma_{p}^{-u}$. Then each cycle of $\tilde{\pi}$ is contained in a cycle of $\tilde{\sigma}$, and for each cycle of $\tilde{\sigma}$ the enclosed cycles of $\tilde{\pi}$ form a non-crossing partition. Thus $\tilde{\pi}$ is non-crossing and $\tilde{\pi} \leq \tilde{\sigma}$. Hence $\pi \in(N C(p) \times N C(q)) \cup S_{N C}(p, q)$. This discussion shows that the partial order $\leq$ could also have been defined as

(i) each cycle of $\pi$ is contained in some cycle of $\sigma$, and

(ii) whenever we have integers $u$ and $v$ such that $\gamma_{p}^{u} \gamma_{q}^{v} \sigma \gamma_{q}^{-v} \gamma_{p}^{-u} \in N C(p+q)$ we also have $\gamma_{p}^{u} \gamma_{q}^{v} \pi \gamma_{q}^{-v} \gamma_{p}^{-u} \in N C(p+q)$.

Lemma 19. Suppose $\pi, \sigma \in S_{N C}(p, q)$ with $\pi \leq \sigma^{-1} \gamma_{p, q}$. Then $\sigma \leq \gamma_{p, q} \pi^{-1}$.

Proof. Since $\pi, \sigma \in S_{N C}(p, q)$ we have $|\pi|+\left|\pi^{-1} \gamma_{p, q}\right|=\left|\gamma_{p, q}\right|+2$ and $|\sigma|+\left|\sigma^{-1} \gamma_{p, q}\right|=$ $\left|\gamma_{p, q}\right|+2$. By hypothesis we have $|\pi|+\left|\pi^{-1} \sigma^{-1} \gamma_{p, q}\right|=\left|\sigma^{-1} \gamma_{p, q}\right|$. Thus

$$
\begin{aligned}
|\sigma|+\left|\sigma^{-1} \gamma_{p, q} \pi^{-1}\right| & =\left|\gamma_{p, q}\right|+2-\left|\sigma^{-1} \gamma_{p, q}\right|+\left|\sigma^{-1} \gamma_{p, q}\right|-|\pi| \\
& =\left|\gamma_{p, q}\right|+2-|\pi|=\left|\pi^{-1} \gamma_{p, q}\right|=\left|\gamma_{p, q} \pi^{-1}\right| .
\end{aligned}
$$

In 6. Definition 4.9] we defined a product for partitioned permutations which we shall recall here. If $\mathcal{V}$ is a partition of $[n]$, then $|\mathcal{V}|=n-\#(\mathcal{V})$, where $\#(\mathcal{V})$ is the number of blocks of $\mathcal{V}$. If $(\mathcal{V}, \pi)$ is a partitioned permutation, then $|(\mathcal{V}, \pi)|=2|\mathcal{V}|-$ $|\pi|$. If $(\mathcal{V}, \pi)$ and $(\mathcal{U}, \sigma)$ are partitioned permutations, then they have a product if $|(\mathcal{V}, \pi)|+|(\mathcal{U}, \sigma)|=|(\mathcal{V} \vee \mathcal{U}, \pi \sigma)|$, and we write this as $(\mathcal{V}, \pi) \cdot(\mathcal{U}, \sigma)=(\mathcal{V} \vee \mathcal{U}, \pi \sigma)$ otherwise the product is not defined.

If $\sigma \in S_{p+q}$ and $\sigma$ has a cycle that connects the two cycles of $\gamma_{p, q}$, then $\sigma \in S_{N C}(p, q)$ if and only if $|\sigma|+\left|\sigma^{-1} \gamma_{p, q}\right|=\left|\gamma_{p, q}\right|+2$. In terms of partitioned permutations this means $\left(0_{\sigma}, \sigma\right) \cdot\left(0_{\sigma^{-1} \gamma_{p, q}}, \sigma^{-1} \gamma_{p, q}\right)=\left(1_{p+q}, \gamma_{p, q}\right)$, as requiring $1_{p+q}=0_{\sigma} \vee 0_{\sigma^{-1} \gamma_{p, q}}$ means that $\sigma$ has a cycle that connects the two cycles of $\gamma_{p, q}$.

Lemma 20. Suppose that $\pi \in N C(p) \times N C(q)$ and $\sigma \in S_{N C}(p, q)$ with $\pi \leq \sigma^{-1} \gamma_{p, q}$. Then

$$
\left(0_{\sigma}, \sigma\right) \cdot\left(0_{\sigma^{-1} \gamma_{p, q} \pi^{-1}}, \sigma^{-1} \gamma_{p, q} \pi^{-1}\right)=\left(\sigma \vee \gamma_{p, q} \pi^{-1}, \gamma_{p, q} \pi^{-1}\right) .
$$

Proof. Since $\pi \leq \sigma^{-1} \gamma_{p, q}$ we have $|\pi|+\left|\pi^{-1} \sigma^{-1} \gamma_{p, q}\right|=\left|\sigma^{-1} \gamma_{p, q}\right|$. Since $\pi \in$ $N C(p) \times N C(q)$ we have $|\pi|+\left|\pi^{-1} \gamma_{p, q}\right|=\left|\gamma_{p, q}\right|$, and since $\sigma \in S_{N C}(p, q)$ we have $|\sigma|+\left|\sigma^{-1} \gamma_{p, q}\right|=\left|\gamma_{p, q}\right|+2$. Thus

$$
\begin{gathered}
\left|\left(0_{\sigma}, \sigma\right)\right|+\left|\left(0_{\sigma^{-1}} \gamma_{p . q} \pi^{-1}, \sigma^{-1} \gamma_{p, q} \pi^{-1}\right)\right|=|\sigma|+\left|\sigma^{-1} \gamma_{p, q} \pi^{-1}\right| \\
=\left|\gamma_{p, q}\right|+2-\left|\sigma^{-1} \gamma_{p, q}\right|+\left|\sigma^{-1} \gamma_{p, q}\right|-|\pi|=\left|\gamma_{p, q} \pi^{-1}\right|+2
\end{gathered}
$$

and also

$$
\left|\left(\sigma \vee \gamma_{p, q} \pi^{-1}, \gamma_{p, q} \pi^{-1}\right)\right|=2\left|\sigma \vee \gamma_{p, q} \pi^{-1}\right|-\left|\gamma_{p, q} \pi^{-1}\right|,
$$

so we must show that $\left|\sigma \vee \gamma_{p, q} \pi^{-1}\right|=\left|\gamma_{p, q} \pi^{-1}\right|+1$, i.e. that $\sigma \vee \gamma_{p, q} \pi^{-1}$ joins two cycles of $\gamma_{p, q} \pi^{-1}$ and the cycles lie on different circles. To achieve this we shall show that if we write $\pi=\pi_{1} \times \pi_{2} \in N C(p) \times N C(q)$, then all through cycles of $\sigma$ meet only one cycle of $\gamma_{p} \pi_{1}^{-1}$. 

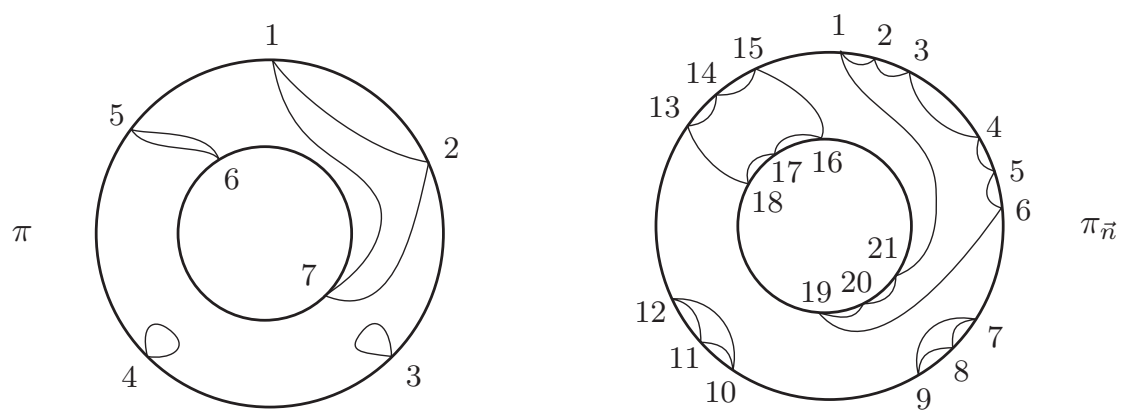

Figure 7. $\pi=(1,2,6)(3)(4)(5,6) \in S_{N C}(5,2)$, all $n_{i}$ 's equal to 3 , and $\pi_{\vec{n}}=(1,2,3,4,5,6,19,20,21)(7,8,9)(10,11,12)(13,14$, $15,16,17,18)$.

Indeed, suppose that two cycles $c$ and $c^{\prime}$ of $\gamma_{p} \pi_{1}^{-1}$ meet through blocks of $\sigma$. Let $a, b \in c$ be respectively the first and last elements of $c$ (since the cycles do not cross, this is well defined). Then $a$ and $b$ are in the same cycle of $\gamma_{p, q} \pi^{-1}$. Thus $\left(\gamma_{p, q}^{-1}(a), b\right) \leq \pi \leq \sigma^{-1} \gamma_{p, q}$. Hence either

(i) as a partition $\sigma \leq\left\{\left(a, \gamma_{p, q}(a), \ldots, b, p+1, \ldots, p+q\right),\left(\gamma_{p, q}(b), \ldots, \gamma_{p, q}^{-1}(a)\right)\right\}$ or

(ii) as a partition $\sigma \leq\left\{\left(a, \gamma_{p, q}(a), \ldots, b\right),\left(\gamma_{p, q}(b), \ldots, \gamma_{p, q}^{-1}(a), p+1, \ldots, p+q\right)\right\}$,

depending on whether the through blocks of $\sigma$ meet the cyclic interval $(a, \ldots, b)$ or the cyclic interval $\left(\gamma_{p, q}(b), \ldots, \gamma_{p, q}^{-1}(a)\right)$. Since $c \subset(a, b)$ and $c^{\prime} \subset\left(\gamma_{p, q}(b), \ldots, \gamma_{p, q}^{-1}(a)\right)$ it is impossible for both $c$ and $c^{\prime}$ to meet through blocks of $\sigma$.

Corollary 21. Given the hypotheses of Lemma 20 we have

(i) every cycle of $\sigma$, which is not a through cycle, is contained in a cycle of $\gamma_{p, q} \pi^{-1}$

(ii) there exist two cycles of $\gamma_{p, q} \pi^{-1}$, one from each circle, such that all the through cycles of $\sigma$ are contained in the union of the supports of these two cycles;

(iii) for each cycle of $\gamma_{p, q} \pi^{-1}$ that does not meet a through cycle of $\sigma$, the enclosed cycles of $\sigma$ form a non-crossing permutation of this cycle of $\gamma_{p, q} \pi^{-1}$;

$(i v)$ the through cycles of $\sigma$ form a non-crossing annular permutation of the union of the two cycles of $\gamma_{p, q} \pi^{-1}$ in (ii) above.

Notation 22. Let $n_{1}, n_{2}, \ldots, n_{r+s}$ be positive integers and let $p=n_{1}+n_{2}+\cdots+n_{r}$ and $q=n_{r+1}+\cdots+n_{r+s}$. Let $N=\left\{n_{1}, n_{1}+n_{2}, \ldots, n_{1}+\cdots+n_{r+s}\right\}$. Given $\pi \in S_{r+s}$ we define $\pi_{\vec{n}} \in S_{p+q}$ as was done in Definition 96 for $i \notin N \pi_{\vec{n}}(i)=i+1$ and $\pi_{\vec{n}}\left(n_{1}+\cdots+n_{k}\right)=n_{1}+\cdots+n_{\pi(k)-1}+1=n_{1}+\cdots+n_{\gamma_{r+s}^{-1} \pi(k)}+1$. Then $\pi_{\vec{n}}^{-1} \gamma_{p, q}\left(n_{1}+\cdots+n_{k}\right)=n_{1}+\cdots+n_{\pi^{-1} \gamma_{r, s}(k)}$. 


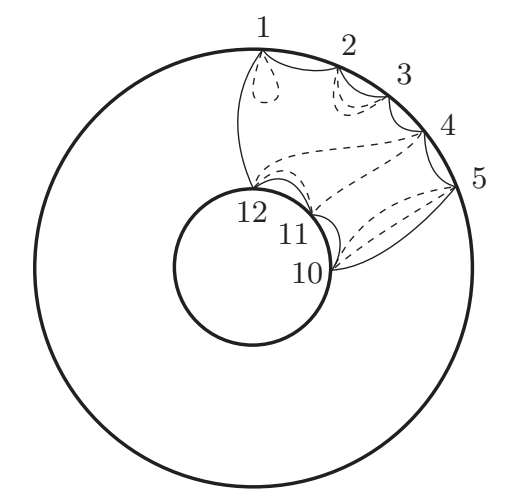

Figure 8. A cycle of a non-crossing annular permutation $\pi$ and, in dotted lines, the enclosed cycles of a $\sigma$ with $\sigma \leq \pi$.

Lemma 23. If $\pi \in S_{N C}(r, s)$, then $\pi_{\vec{n}} \in S_{N C}(p, q)$.

Proof. Let $\psi:[r+s] \rightarrow[p+q]$ be given by $\psi(i)=n_{1}+\cdots+n_{i}$. Then as in Lemma 12 we have $\psi \pi^{-1} \gamma_{r, s}=\pi_{\vec{n}}^{-1} \gamma_{p, q} \psi$. Thus

$$
\begin{aligned}
& \#\left(\pi_{\vec{n}}\right)+\#\left(\pi_{\vec{n}}^{-1} \gamma_{p, q}\right)+\#\left(\gamma_{p, q}\right) \\
& \quad=\#(\pi)+\#\left(\pi^{-1} \gamma_{r, s}\right)+p+q-(r+s)+\#\left(\gamma_{r, s}\right)=p+q+2 .
\end{aligned}
$$

Proposition 24. Let $\pi \in S_{N C}(r, s)$ and $\pi_{\vec{n}} \in S_{N C}(p, q)$. If $\sigma \in S_{p+q}$ is such that

(i) each cycle of $\sigma$ is contained in some cycle of $\pi_{\vec{n}}$,

(ii) for each cycle of $\pi_{\vec{n}}$ the enclosed cycles of $\sigma$ form a non-crossing permutation (see Figure 8), and

(iii) for each cycle $\tilde{c}_{i}$ of $\pi_{\vec{n}}$ we have $\sigma_{i} \vee \tau_{i}=\tilde{c}_{i}$, where $\sigma_{i}$ and $\tau_{i}$ are the respective restrictions of $\sigma$ and $\tau_{\vec{n}}$ to $\tilde{c}_{i}$,

then $\sigma \in S_{N C}(p, q)$ and $\sigma^{-1} \pi_{\vec{n}}$ separates the points of $N$.

Proof. From the remark above $\sigma \in(N C(p) \times N C(q)) \cup S_{N C}(p, q)$. From the theorem above $\sigma^{-1} \pi_{\vec{n}}$ separates the points of $N$. Since $\pi$ has a through block (i.e. a cycle that connects the two circles) there are $k_{1}, l_{2} \leq p$ and $k_{2}, l_{1}>p$ all in the same cycle of $\pi$, so that $\pi\left(k_{1}\right)=l_{1}$ and $\pi\left(k_{2}\right)=l_{2}$. Then $\pi_{\vec{n}}\left(n_{1}+\cdots+n_{k_{1}}\right)=n_{1}+\cdots+n_{l_{1}-1}+1$ and $\pi_{\vec{n}}\left(n_{1}+\cdots+n_{k_{2}}\right)=n_{1}+\cdots+n_{l_{2}-1}+1$. Let $\tilde{c}_{i}$ be the cycle of $\pi_{\vec{n}}$ containing $n_{1}+\cdots+n_{k_{1}}$ and hence $n_{1}+\cdots+n_{k_{2}}$. Since $\sigma^{-1} \tilde{c}_{i}$ separates the points of $N \cap \tilde{c}_{i}$, $n_{1}+\cdots+n_{k_{1}}$ and $n_{1}+\cdots+n_{k_{2}}$ must be in different cycles of $\sigma^{-1} \tilde{c}_{i}$. Hence by Lemma 13, $\sigma_{i} \not \leq \tilde{c}_{i}\left(n_{1}+\cdots+n_{k_{1}}, n_{1}+\cdots+n_{k_{2}}\right)=d_{1} d_{2}$, where the points on the cycle $d_{1}$ are contained in $[p]$ and those of $d_{2}$ in $[p+1, p+q]=\{p+1, \ldots, p+q\}$. Thus $\sigma_{i}$ has a through block, and so $\sigma \in S_{N C}(p, q)$.

Our last step in this section is to translate the criterion of equation (44) to the annular case. We suppose we have $a_{1}, \ldots, a_{p+q} \in \mathcal{A}$ and we let $A_{1}=a_{1} \cdots a_{n_{1}}, \ldots$, $A_{r+s}=a_{n_{1}+\cdots+n_{r+s-1}+1} \cdots a_{n_{1}+\cdots+n_{r+s}}$. 
Proposition 25. Let $\pi \in S_{N C}(r, s)$ and $\pi_{\vec{n}} \in S_{N C}(p, q)$. Then

$$
\kappa_{\pi}\left(A_{1}, \ldots, A_{r+s}\right)=\sum_{\sigma \in S_{N C}(p, q)} \kappa_{\sigma}\left(a_{1}, \ldots, a_{p+q}\right),
$$

where the sum is over all $\sigma$ such that $\sigma^{-1} \pi_{\vec{n}}$ separates the points of $N$.

Proof. Let $c_{1} c_{2} \cdots c_{k}$ be the cycle decomposition of $\pi$ and $\tilde{c}_{1} \tilde{c}_{2} \cdots \tilde{c}_{k}$ the corresponding cycle decomposition of $\pi_{\vec{n}}$, as in the proof of Theorem 15. Using the notation of equation (2)

$$
\kappa_{\pi}\left(A_{1}, \ldots, A_{r+s}\right)=\kappa_{c_{1}}\left(A_{1}, \ldots, A_{r+s}\right) \cdots \kappa_{c_{k}}\left(A_{1}, \ldots, A_{r+s}\right) .
$$

By equation (4)

$$
\kappa_{c_{i}}\left(A_{1}, \ldots, A_{r+s}\right)=\sum_{\sigma_{i}} \kappa_{\sigma_{i}}\left(a_{1}, \ldots, a_{p+q}\right),
$$

where $\sigma_{i}$ runs over all non-crossing partitions of the point set of the cycle $\tilde{c}_{i}$ such that $\sigma_{i} \vee \tau_{i}=\tilde{c}_{i}$ (using the notation of Theorem [15), or equivalently that $\sigma_{i}^{-1} \tilde{c}_{i}$ separates the points of $N \cap \tilde{c}_{i}$. Thus

$$
\kappa_{\pi}\left(A_{1}, \ldots, A_{r+s}\right)=\sum_{\sigma_{1}} \cdots \sum_{\sigma_{k}} \kappa_{\sigma_{1}}\left(a_{1}, \ldots, a_{p+q}\right) \cdots \kappa_{\sigma_{k}}\left(a_{1}, \ldots, a_{p+q}\right),
$$

where for each $i, \sigma_{i}$ runs over the non-crossing partitions of $\tilde{c}_{i}$ such that $\sigma_{i}^{-1} \tilde{c}_{i}$ separates the points of $N \cap \tilde{c}_{i}$. Multiplying the $\sigma_{i}$ 's together we obtain all the $\sigma$ 's satisfying the hypotheses of Proposition 24. Thus the sum is over all $\sigma \in S_{N C}(p, q)$ such that $\sigma^{-1} \pi_{\vec{n}}$ separates the points of $N$.

\section{Proof of the MAIN THEOREM}

Throughout this section we shall assume that we are given positive integers $n_{1}, n_{2}, \ldots, n_{r+s}$ and elements $a_{1}, \ldots, a_{n_{1}+\cdots+n_{r+s}} \in \mathcal{A}$, where $\left(\mathcal{A}, \phi, \phi_{2}\right)$ is a second order non-commutative probability space. We let $N=\left\{n_{1}, n_{1}+n_{2}, \ldots, n_{1}+\cdots+\right.$ $\left.n_{r+s}\right\}, p=n_{1}+\cdots+n_{r}, q=n_{r+1}+\cdots+n_{r+s}$, and $N_{1}=[p] \cap N$ and $N_{2}=N \cap$ $[p+1, p+q]$. We let $A_{1}=a_{1} \cdots a_{n_{1}}, \ldots, A_{r+s}=a_{n_{1}+\cdots+n_{r+s-1}+1} \cdots a_{n_{1}+\cdots+n_{r+s}}$.

We shall prove Theorem 3 by induction on $(r, s)$. So to begin let us prove the theorem when $r=s=1$.

\section{Lemma 26.}

$$
\begin{aligned}
\kappa_{1,1}\left(a_{1} \cdots a_{p}, a_{p+1} \cdots a_{p+q}\right)= & \sum_{\sigma \in S_{N C}(p, q)} \kappa_{\sigma}\left(a_{1}, \ldots, a_{p+q}\right) \\
& +\sum_{(\mathcal{U}, \sigma)} \kappa_{(\mathcal{U}, \sigma)}\left(a_{1}, \ldots, a_{p+q}\right),
\end{aligned}
$$

where in the first sum $\sigma$ is such that $p$ and $p+q$ are in different cycles of $\sigma^{-1} \gamma_{p, q}$ and in the second sum $(\mathcal{U}, \sigma)$ runs over $\mathcal{P S}_{N C}(p, q)^{\prime}$.

Proof. We have two expressions for $\phi_{2}\left(a_{1} \cdots a_{p}, a_{p+1} \cdots a_{p+q}\right)$. The first is

$$
\begin{aligned}
\phi_{2}\left(a_{1} \cdots a_{p}, a_{p+1} \cdots a_{p+q}\right)= & \sum_{\sigma \in S_{N C}(p, q)} \kappa_{\sigma}\left(a_{1}, \ldots, a_{p+q}\right) \\
& +\sum_{(\mathcal{U}, \sigma) \in \mathcal{P} \mathcal{S}_{N C}(p, q)^{\prime}} \kappa_{(\mathcal{U}, \sigma)}\left(a_{1}, \ldots, a_{p+q}\right),
\end{aligned}
$$


and the second is

$$
\begin{aligned}
\phi_{2}\left(a_{1} \cdots a_{p}, a_{p+1} \cdots a_{p+q}\right)= & \kappa_{2}\left(a_{1} \cdots a_{p}, a_{p+1} \cdots a_{p+q}\right) \\
& +\kappa_{1,1}\left(a_{1} \cdots a_{p}, a_{p+1} \cdots a_{p+q}\right) .
\end{aligned}
$$

Combining these two with equation (4) we have

$$
\begin{aligned}
\kappa_{1,1}\left(a_{1} \cdots a_{p}, a_{p+1} \cdots a_{p+q}\right) & =\sum_{\sigma \in S_{N C}(p, q)} \kappa_{\sigma}\left(a_{1}, \ldots, a_{p+q}\right) \\
& -\sum_{\sigma \in N C(p+q)} \kappa_{\sigma}\left(a_{1}, \ldots, a_{p+q}\right)+\sum_{(\mathcal{U}, \sigma)} \kappa_{(\mathcal{U}, \sigma)}\left(a_{1}, \ldots, a_{p+q}\right),
\end{aligned}
$$

where the second sum, by Lemma 14, is over $\sigma \in N C(p+q)$ such that $p$ and $p+q$ are in different cycles of $\sigma^{-1} \gamma_{p+q}$.

Now each $\sigma \in N C(p+q)$ such that $p$ and $p+q$ are in different cycles of $\sigma^{-1} \gamma_{p+q}$ must have a through block by Lemma 13 (i.e. a block connecting a point in $[p]$ to one in $[p+1, p+q])$ and thus by [20, Equation 5.2] is a also a non-crossing $(p, q)$ annular permutation. Finally since $\sigma^{-1} \gamma_{p, q}=\sigma^{-1} \gamma_{p+q}(p, p+q)$, we have that $p$ and $p+q$ are in the same cycle of $\sigma^{-1} \gamma_{p, q}$. Hence the $\sigma$ 's in the second sum are exactly those in $S_{N C}(p, q)$ for which $p$ and $p+q$ are in the same cycle of $\sigma^{-1} \gamma_{p, q}$. Removing these from the first sum we are left with the $\sigma$ 's for which $p$ and $p+q$ are in different cycles of $\sigma^{-1} \gamma_{p, q}$.

Note that for the term

$$
\sum_{(\mathcal{U}, \sigma)} \kappa_{(\mathcal{U}, \sigma)}\left(a_{1}, \ldots, a_{p+q}\right)
$$

$\sigma=\sigma_{1} \times \sigma_{2} \in N C(p) \times N C(q)$, so that the condition that $p$ and $p+q$ are in different cycles of $\sigma^{-1} \gamma_{p, q}=\sigma_{1}^{-1} \gamma_{p} \times \sigma_{2} \gamma_{q}$ is automatically satisfied.

In order to give an inductive proof of our main theorem we shall need an extension of its statement to the case of partitioned permutations.

Lemma 27. Suppose that equation (9) holds for $r^{\prime} \leq r$ and $s^{\prime} \leq s$. Then for $(\mathcal{V}, \pi) \in \mathcal{P} \mathcal{S}_{N C}(r, s)^{\prime}$ we have

$$
\kappa_{(\mathcal{V}, \pi)}\left(A_{1}, \ldots, A_{r+s}\right)=\sum_{\sigma \in S_{N C}(p, q)} \kappa_{\sigma}\left(a_{1}, \ldots, a_{p+q}\right)+\sum_{(\mathcal{U}, \sigma)} \kappa_{(\mathcal{U}, \sigma)}\left(a_{1}, \ldots, a_{p+q}\right),
$$

where the first sum is over all $\sigma$ 's such that $\sigma \leq \mathcal{V}_{\vec{n}}$ (i.e. each cycle of $\sigma$ is contained in a block of $\mathcal{V}_{\vec{n}}$ ) and $\sigma^{-1} \pi_{\vec{n}}$ separates the points of $N$, and the second sum is over all $(\mathcal{U}, \sigma)$ such that $\mathcal{U} \leq \mathcal{V}_{\vec{n}}$ and $\sigma^{-1} \pi_{\vec{n}}$ separates the points of $N$.

Proof. Let us write $\pi$ as a product of cycles: $c_{1} \cdots c_{k} c^{\prime} c^{\prime \prime}$, where the cycles $c_{1}, \ldots$, $c_{k}$ are the cycles of $\pi$ that are the only cycles in the block of $\mathcal{V}$ that contain them and $c^{\prime}$ and $c^{\prime \prime}$ are the two cycles of $\pi$ that lie in a single block of $\mathcal{V}$. Let $1_{c^{\prime} \cup c^{\prime \prime}}$ be this block of $\mathcal{V}$ that contains the two cycles $c^{\prime}$ and $c^{\prime \prime}$. Then by equation (9)

$$
\begin{aligned}
\kappa_{(\mathcal{V}, \pi)} & \left(A_{1}, \ldots, A_{r+s}\right)=\kappa_{c_{1}}\left(A_{1}, \ldots, A_{r+a}\right) \cdots \kappa_{c_{k}}\left(A_{1}, \ldots, A_{r+s}\right) \\
& \times \kappa_{\left(1_{\left.c^{\prime} \cup c^{\prime \prime}, c^{\prime} c^{\prime \prime}\right)}\left(A_{1}, \ldots, A_{r+s}\right)\right.}{ } \sum_{\sigma_{1}, \ldots, \sigma_{k}} \kappa_{\sigma_{1}}\left(a_{1}, \ldots, a_{p+q}\right) \cdots \kappa_{\sigma_{k}}\left(a_{1}, \ldots, a_{p+q}\right) \\
& \times\left\{\sum_{\sigma_{0}} \kappa_{\sigma_{0}}\left(a_{1}, \ldots, a_{p+q}\right)+\sum_{\left(\mathcal{U}_{0}, \sigma_{00}\right)} \kappa_{\left(\mathcal{U}_{0}, \sigma_{00}\right)}\left(a_{1}, \ldots, a_{p+q}\right)\right\},
\end{aligned}
$$


where $\sigma_{i}$ runs over $N C\left(\tilde{c}_{i}\right)$ such that $\sigma^{-1} \tilde{c}_{i}$ separates the points of $N \cap \tilde{c}_{i} ; \sigma_{0}$ runs over $S_{N C}\left(\tilde{c}^{\prime}, \tilde{c}^{\prime \prime}\right)$ such that $\sigma_{0}^{-1} \tilde{c}^{\prime} \tilde{c}^{\prime \prime}$ separates the points of $N \cap 1_{\tilde{c}^{\prime} \cup \tilde{c}^{\prime \prime}}$; and $\left(\mathcal{U}_{0}, \sigma_{00}\right)$ runs over $\mathcal{P} \mathcal{S}_{N C}\left(\tilde{c}^{\prime}, \tilde{c}^{\prime \prime}\right)$ such that $\sigma_{00}^{-1} \tilde{c}^{\prime} \tilde{c}^{\prime \prime}$ separates the points of $N \cap 1_{\tilde{c}^{\prime} \cup \tilde{c}^{\prime \prime}}$. The product $\sigma_{0} \sigma_{1} \cdots \sigma_{k}$ thus runs over $\sigma \in S_{N C}(p, q)$ such that $\sigma^{-1} \pi_{\vec{n}}$ separates the points of $N$ and $\sigma \leq \mathcal{V}_{\vec{n}}$. The product $\left(\mathcal{U}_{0}, \sigma_{00}\right) \sigma_{1} \cdots \sigma_{k}$ runs over $(\mathcal{U}, \sigma) \in$ $\mathcal{P} \mathcal{S}_{N C}(p, q)^{\prime}$ such that $\sigma^{-1} \pi_{\vec{n}}$ separates the points of $N$ and $\mathcal{U} \leq \mathcal{V}_{\vec{n}}$. This proves our assertion.

In the next lemma we consider $\sigma \in S_{N C}(p, q)$ such that $\sigma^{-1} \gamma_{p, q}$ fails to separate the points of $N$. We wish to determine $\pi \in S_{N C}(r, s)$ such that $\pi_{\vec{n}}$ will behave like the supremum of $\sigma$ and $\tau_{\vec{n}}$ in that with respect to $\pi_{\vec{n}}, \sigma$ will satisfy the three conditions of Proposition 24

Lemma 28. Let $\sigma \in S_{N C}(p, q)$ be such that $\sigma^{-1} \gamma_{p, q}$ has a cycle that meets both $N_{1}=N \cap[n]$ and $N_{2}=N \cap[p+1, p+q]$. Then there is a unique $\pi \in S_{N C}(r, s)$ such that relative to $\pi, \sigma$ satisfies the three conditions of Proposition 24,

Proof. Let us first deal with uniqueness. Suppose $\pi \in S_{N C}(r, s)$ is such that $\sigma^{-1} \pi_{\vec{n}}$ separates the points of $N$. Then $\left.\left(\sigma^{-1} \pi_{\vec{n}}\right)\right|_{N}=i d_{N}$ and for $i \notin N, \pi_{\vec{n}}^{-1} \gamma_{p, q}(i)=i$. Thus by Lemma 6. $\left.\left(\sigma^{-1} \gamma_{p, q}\right)\right|_{N}=\left.\left(\sigma^{-1} \pi_{\vec{n}}\right)\right|_{N} \cdot\left(\pi_{\vec{n}}^{-1} \gamma_{p, q}\right)\left|N=\left(\pi_{\vec{n}}^{-1} \gamma_{p, q}\right)\right|_{N}$. Since $\pi_{\vec{n}}^{-1} \gamma_{p, q} \psi=\psi \pi^{-1} \gamma_{r, s}$ by Lemma 23, $\pi$ is uniquely determined by $\left.\left(\sigma^{-1} \gamma_{p, q}\right)\right|_{N}$.

To show existence consider $\left.\left(\sigma^{-1} \gamma_{p, q}\right)\right|_{N}$. By Lemma 6 .

$$
\left.\left(\sigma^{-1} \gamma_{p, q}\right)\right|_{N} \in S_{N C}\left(N_{1}, N_{2}\right) \cup\left(N C\left(N_{1}\right) \times N C\left(N_{2}\right)\right) .
$$

By assumption, $\sigma^{-1} \gamma_{p, q}$ has a cycle meeting both $N_{1}$ and $N_{2}$; thus $\left.\left(\sigma^{-1} \gamma_{p, q}\right)\right|_{N}$ $\in S_{N C}\left(N_{1}, N_{2}\right)$. Then there is $\pi \in S_{N C}(r, s)$ such that $\psi \pi^{-1} \gamma_{r, s} \psi^{-1}=$ $\left.\left(\sigma^{-1} \gamma_{p, q}\right)\right|_{N}$.

Now $\pi_{\vec{n}}^{-1} \gamma_{p, q}(i)=i$ for $i \notin N$ and $\left.\pi_{\vec{n}}^{-1} \gamma_{p, q}\right|_{N}=\left.\sigma^{-1} \gamma_{p, q}\right|_{N}$ together imply that $\pi_{\vec{n}}^{-1} \gamma_{p, q} \leq \sigma^{-1} \gamma_{p, q}$, and thus by Lemma 19, $\sigma \leq \pi_{\vec{n}}$ and the cycles of $\sigma$ form a non-crossing partition of those of $\pi_{\vec{n}}$. Finally by applying Lemma 14 to each cycle of $\pi_{\vec{n}}$ we obtain that the three conditions of Proposition[24 are satisfied. See Figure 9.

Lemma 29. Suppose that $\sigma \in S_{N C}(p, q)$ is such that $\sigma^{-1} \gamma_{p, q}$ does not separate the points of $N$ but no cycle of $\sigma^{-1} \gamma_{p, q}$ meets both $N_{1}$ and $N_{2}$. Then there is a unique $(\mathcal{V}, \pi) \in \mathcal{P} \mathcal{S}_{N C}(r, s)$ such that $\mathcal{V}_{\vec{n}}=\sigma \vee \tau_{\vec{n}}$ and $\sigma^{-1} \pi_{\vec{n}}$ separates the points of $N$.

Proof. Let us first deal with uniqueness. $\mathcal{V}_{\vec{n}}$ is determined by the equation $\mathcal{V}_{\vec{n}}=$ $\sigma \vee \tau_{\vec{n}}$. If $\sigma^{-1} \pi$ separates the points of $N$, then $\left.\sigma^{-1} \pi_{\vec{n}}\right|_{N}=i d_{N}$. Also $\pi_{\vec{n}}^{-1} \gamma_{p, q}$ is the identity on $N^{c}$; thus by Lemma $\left.6 \sigma^{-1} \gamma_{p, q}\right|_{N}=\left.\left.\sigma^{-1} \pi_{\vec{n}}\right|_{N} \cdot \pi_{\vec{n}}^{-1} \gamma_{p, q}\right|_{N}=\left.\pi_{\vec{n}}^{-1} \gamma_{p, q}\right|_{N}$. Since $\pi_{\vec{n}}^{-1} \gamma_{p, q} \psi=\psi \pi^{-1} \gamma_{r, s}$ we see that $\pi$ is uniquely determined.

Secondly let us prove existence. Let us denote by $N C\left(N_{1}\right)$ and $N C\left(N_{2}\right)$ respectively the non-crossing partitions of $N_{1}$ and $N_{2}$ where the order is that determined by $\gamma_{p, q}$. Similarly $S_{N C}\left(N_{1}, N_{2}\right)$ denotes the non-crossing annular permutations on $N_{1} \cup N_{2}$, again with the order determined by $\gamma_{p, q}$.

By Lemma 5

$$
\left.\sigma^{-1} \gamma_{p, q}\right|_{N} \in S_{N C}\left(N_{1}, N_{2}\right) \cup\left(N C\left(N_{1}\right) \times N C\left(N_{2}\right)\right) .
$$

By assumption no cycle $\left.\sigma^{-1} \gamma_{p, q}\right|_{N}$ meets both $N_{1}$ and $N_{2}$, so $\left.\sigma^{-1} \gamma_{p, q}\right|_{N} \in N C\left(N_{1}\right) \times$ $N C\left(N_{2}\right)$. Thus there is $\pi \in N C(r) \times N C(s)$ such that $\psi \pi^{-1} \gamma_{r, s} \psi^{-1}=\left.\sigma^{-1} \gamma_{p, q}\right|_{N}$, 

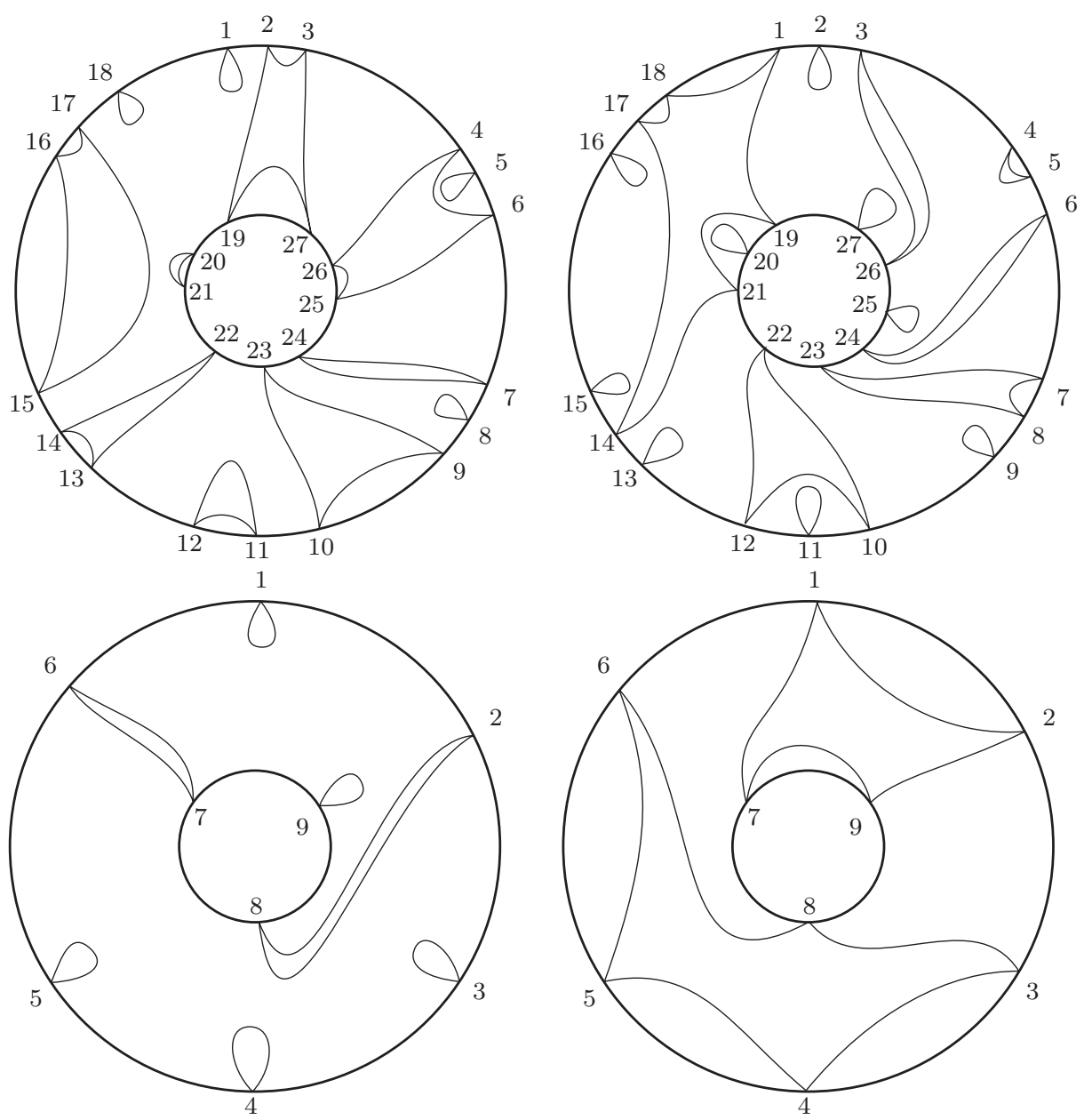

Figure 9. Shown is $\sigma \in S_{N C}(18,9)$ in the upper left, $\sigma^{-1} \gamma_{18,9}$ in the upper right. In the lower left we have $\pi^{-1} \gamma_{6,3}$ and in the lower right we have $\pi \in S_{N C}(6,3) . \quad \pi_{\vec{n}}$ is shown overleaf. Note that when $N=\{3,6,9,12,15,18,21,24,27\}, \sigma$ satisfies the hypotheses of Lemma 28 for example 6 and 24 are in the same cycle of $\left.\sigma^{-1} \gamma_{18,9}\right|_{N}$. The resulting $\pi^{-1} \gamma_{6,3}$ has 2 and 8 in the same cycle and thus $\pi \in S_{N C}(6,3)$.

and thus $\left.\sigma^{-1} \gamma_{p, q}\right|_{N}=\left.\pi_{\vec{n}}^{-1} \gamma_{p, q}\right|_{N}$. By Lemma 6] $\left.\sigma^{-1} \pi_{\vec{n}}\right|_{N}=i d_{N}$, so $\sigma^{-1} \pi_{\vec{n}}$ separates the points of $N$.

Recall that $\pi_{\vec{n}}^{-1} \gamma_{p, q}(i)=i$ for $i \notin N$ and $\left.\pi^{-1} \gamma_{p, q}\right|_{N}=\left.\sigma^{-1} \gamma_{p, q}\right|_{N}$; thus $\pi_{\vec{n}}^{-1} \gamma_{p, q} \leq$ $\sigma^{-1} \gamma_{p, q}$. Therefore, by Corollary 21, $\sigma \vee \pi_{\vec{n}}$ is obtained from the cycles of $\pi_{\vec{n}}$ by joining the two cycles of $\pi_{\vec{n}}$, one from each circle, that meet the through cycles of $\sigma$. To complete the proof we must show that $\sigma \vee \tau_{\vec{n}}=\sigma \vee \pi_{\vec{n}}$; for this we must show that $\pi_{\vec{n}} \leq \sigma \vee \tau_{\vec{n}}$, i.e. that any two blocks of $\tau_{\vec{n}}$ connected by $\pi_{\vec{n}}$ are connected by $\sigma$. 

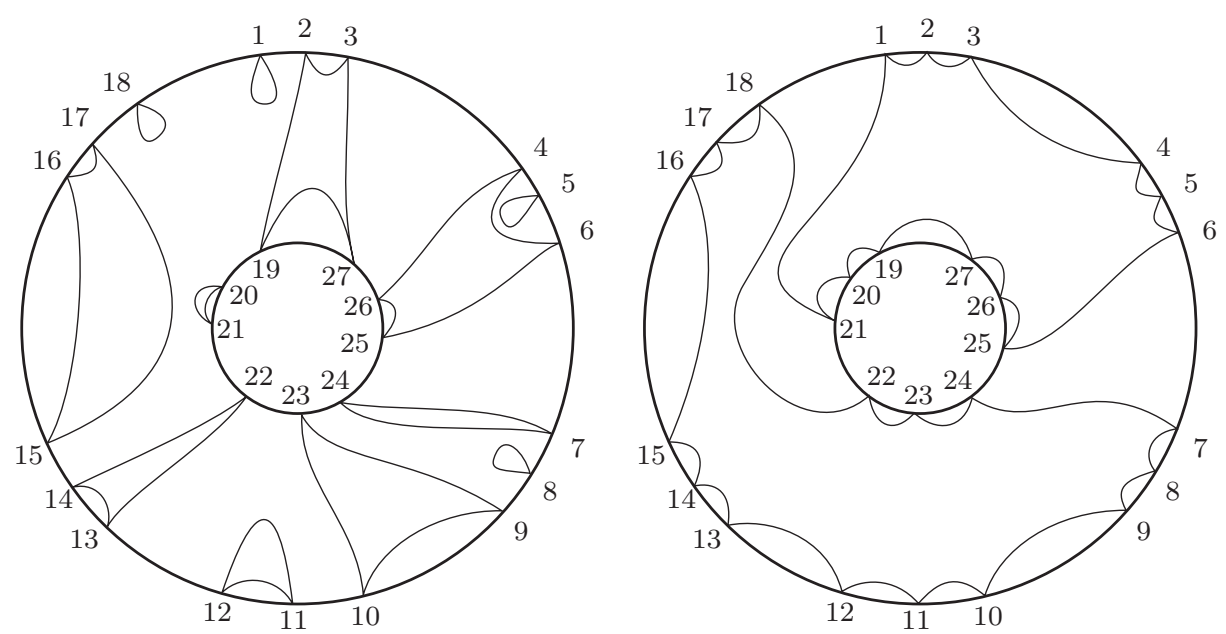

Figure 10. $\sigma$ (left) from Figure 9 is compared with the $\pi_{\vec{n}} \in$ $S_{N C}(18,9)$ produced by Lemma 28 .

Let $c$ be a cycle of $\pi_{\vec{n}}$ not meeting a through cycle of $\sigma$ and let $\tilde{\sigma}$ the product of the cycles of $\sigma$ enclosed by $c$. Then $\tilde{\sigma}^{-1} c$ separates the points of $N$ in $c$, and so by Lemma 14 any two blocks of $\tau_{\vec{n}}$ in $c$ connected by $c$ are connected by $\tilde{\sigma}$.

Now let $c$ and $c^{\prime}$ be the two cycles (one in each cycle of $\gamma_{p, q}$ ) that meet the through cycles of $\sigma$ and let $\tilde{\sigma}$ be the product of the cycles of $\sigma$ enclosed by $c \cup c^{\prime}$ and the same for $\tilde{\tau}$. Now we must show that $\tilde{\sigma} \vee \tilde{\tau}=c \cup c^{\prime}$. If not, then there would be $r \neq s \in N$ such that $(r, s) \leq \tilde{\sigma}^{-1} c c^{\prime}$, in which case $r$ and $s$ are in the same cycle of $\sigma^{-1} \pi_{\vec{n}}$, contrary to our hypothesis. Hence $\sigma \vee \tau_{\vec{n}}=\pi_{\vec{n}}$.

Proof of Theorem 3. Let us write $\phi_{2}\left(A_{1} \cdots A_{r}, A_{r+1} \cdots A_{r+s}\right)$ in two different ways:

$$
\begin{aligned}
\phi_{2}\left(A_{1} \cdots A_{r}, A_{r+1} \cdots A_{r+s}\right)=\sum_{\pi \in S_{N C}(r, s)} \kappa_{\pi}\left(A_{1}, \ldots, A_{r+s}\right) \\
+\sum_{(\mathcal{V}, \pi) \in \mathcal{P} \mathcal{S}_{N C}(p, q)^{\prime}} \kappa_{(\mathcal{V}, \pi)}\left(A_{1}, \ldots, A_{r+s}\right) \\
=\sum_{\pi \in S_{N C}(r, s)} \kappa_{\pi}\left(A_{1}, \ldots, A_{r+s}\right) \\
\quad+\sum_{(\mathcal{V}, \pi) \in \mathcal{P} \mathcal{S}_{N C}(p, q)^{\prime \prime}} \kappa_{(\mathcal{V}, \pi)}\left(A_{1}, \ldots, A_{r+s}\right) \\
\quad+\kappa_{r, s}\left(A_{1}, \ldots, A_{r+s}\right),
\end{aligned}
$$

where $\mathcal{P} \mathcal{S}_{N C}(r, s)^{\prime \prime}=\mathcal{P} \mathcal{S}_{N C}(r, s)^{\prime} \backslash\left\{\left(1_{r+s}, \gamma_{r, s}\right)\right\}$. Also

$$
\begin{aligned}
& \phi_{2}\left(A_{1} \cdots A_{r}, A_{r+1} \cdots A_{r+s}\right)=\phi_{2}\left(a_{1} \cdots a_{p}, a_{p+1} \cdots a_{p+q}\right) \\
& \quad=\sum_{\pi \in S_{N C}(p, q)} \kappa_{\pi}\left(a_{1}, \ldots, a_{p+q}\right)=\sum_{(\mathcal{V}, \pi) \in \mathcal{P} \mathcal{S}_{N C}(p, q)^{\prime}} \kappa_{(\mathcal{V}, \pi)}\left(a_{1}, \ldots, a_{p+q}\right) .
\end{aligned}
$$



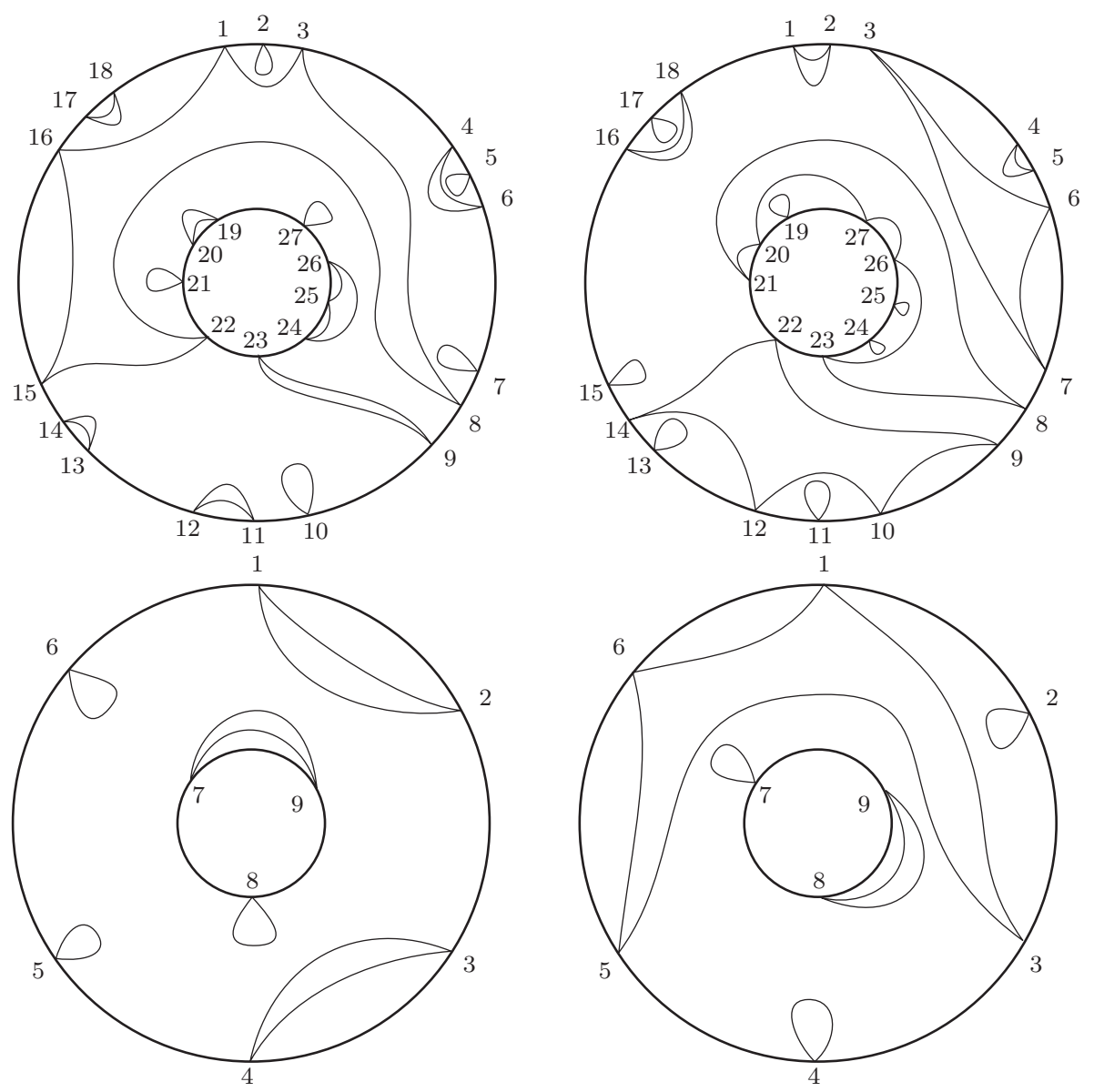

Figure 11. Shown is $\sigma \in S_{N C}(18,9)$ in the upper left, $\sigma^{-1} \gamma_{18,9}$ in the upper right. In the lower left we have $\pi^{-1} \gamma_{6,3}$ and in the lower right we have $\pi \in S_{N C}(6,3) . \pi_{\vec{n}}$ is shown overleaf. Note that when $N=\{3,6,9,12,15,18,21,24,27\}, \sigma$ satisfies the hypotheses of Lemma 29. for example 3 and 6 are in the same cycle of $\left.\sigma^{-1} \gamma_{18,9}\right|_{N}$. The resulting $\pi^{-1} \gamma_{6,3}$ has 1 and 2 in the same cycle.

Now solving for $\kappa_{r, s}\left(A_{1}, \ldots, A_{r+s}\right)$ we have

$$
\begin{aligned}
\kappa_{r, s}\left(A_{1}, \ldots, A_{r+s}\right)= & \sum_{\sigma \in S_{N C}(p, q)} \kappa_{\sigma}\left(a_{1}, \ldots, a_{p+q}\right) \\
& -\sum_{\pi \in S_{N C}(r, s)} \kappa_{\pi}\left(A_{1}, \ldots, A_{r+s}\right) \\
& +\sum_{(\mathcal{V}, \pi) \in \mathcal{P} \mathcal{S}_{N C}(p, q)} \kappa_{(\mathcal{V}, \pi)}\left(a_{1}, \ldots, a_{p+q}\right) \\
& -\sum_{(\mathcal{V}, \pi) \in \mathcal{P} \mathcal{S}_{N C}(r, s)^{\prime \prime}} \kappa_{(\mathcal{V}, \pi)}\left(A_{1}, \ldots, A_{r+s}\right) .
\end{aligned}
$$



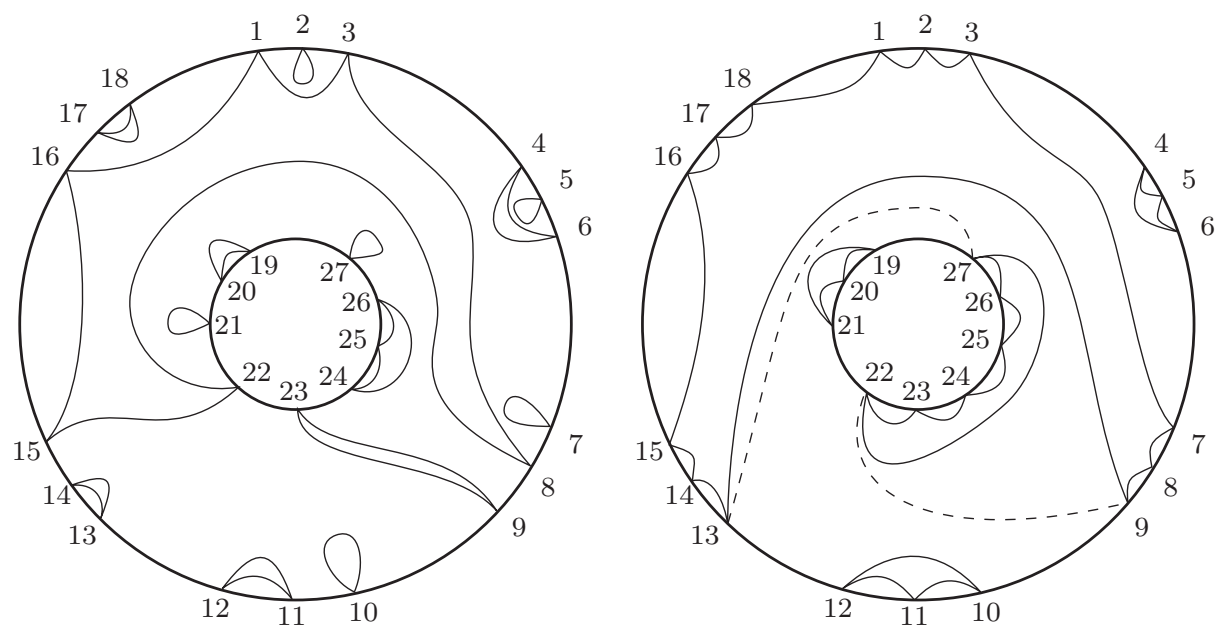

Figure 12. $\sigma$ (left) from Figure 11 is compared with the $\pi_{\vec{n}} \in$ $N C(18) \times N C(9)$ produced by Lemma 29, The two cycles of $\pi_{\vec{n}}$ that meet through cycles of $\sigma$ are shown connected by dotted lines.

Now by Proposition 25

$$
\sum_{\pi \in S_{N C}(r, s)} \kappa_{\pi}\left(A_{1}, \ldots, A_{r+s}\right)=\sum_{\pi \in S_{N C}(r, s)} \sum_{\substack{\sigma \in S_{N C}(p, q) \\ \sigma^{-1} \pi_{\vec{n}} \text { sep.'s } N}} \kappa_{\sigma}\left(a_{1}, \ldots, a_{p+q}\right) .
$$

If $\pi \in S_{N C}(r, s)$ and $\sigma^{-1} \pi_{\vec{n}}$ separates the points of $N$, then $\left.\sigma^{-1} \gamma_{p, q}\right|_{N}=\left.\pi_{\vec{n}}^{-1} \gamma_{p, q}\right|_{N}$ by Lemma 6 and $\pi_{\vec{n}}^{-1} \gamma_{p, q}$ has a cycle that meets both $N_{1}$ and $N_{2}$. Conversely by Lemma 28 if $\sigma \in S_{N C}(p, q)$ is such that $\left.\sigma^{-1} \gamma_{p, q}\right|_{N}$ has a cycle that meets both $N_{1}$ and $N_{2}$, then $\sigma$ comes from a unique $\pi \in S_{N C}(r, s)$. Thus

$$
\begin{gathered}
\sum_{\sigma \in S_{N C}(p, q)} \kappa_{\sigma}\left(a_{1}, \ldots, a_{p+q}\right)-\sum_{\pi \in S_{N C}(r, s)} \sum_{\substack{\sigma \in S_{N C}(p, q) \\
\sigma-1 \pi_{\vec{n}} \operatorname{sep} . ' s ~}} \kappa_{\sigma}\left(a_{1}, \ldots, a_{p+q}\right) \\
=\sum_{\substack{\sigma \in S_{N C}(p, q) \\
\sigma^{-1} \gamma_{p, q} \text { sep.'s } N}} \kappa_{\sigma}\left(a_{1}, \ldots, a_{p+q}\right) .
\end{gathered}
$$

To conclude the proof we use induction on $r$ and $s$. In Lemma 26 we have proved equation (9) when $r=s=1$. We can apply the induction hypothesis to $\mathcal{P} \mathcal{S}_{N C}(r, s)^{\prime \prime}$ because $\left(1_{r+s}, \gamma_{r, s}\right)$ has been removed. Thus by Lemma 27 we have:

$$
\begin{aligned}
& \sum_{(\mathcal{V}, \pi) \in \mathcal{P} \mathcal{S}_{N C}(r, s)^{\prime \prime}} \kappa_{(\mathcal{V}, \pi)}\left(A_{1}, \ldots, A_{r+s}\right) \\
= & \sum_{(\mathcal{V}, \pi) \in \mathcal{P} \mathcal{S}_{N C}(r, s)^{\prime \prime}} \sum_{\substack{(\mathcal{U}, \sigma) \in \mathcal{P} \mathcal{S}_{N C}(p, q)^{\prime} \\
\sigma^{-1} \pi_{\vec{n}} \operatorname{sep} . ' s}} \kappa_{(\mathcal{U}, \sigma)}\left(a_{1}, \ldots, a_{p+q}\right) \\
= & \sum_{\begin{array}{c}
(\mathcal{U}, \sigma) \in \mathcal{P} \mathcal{S}_{N C}(p, q)^{\prime} \\
\sigma^{-1} \gamma_{p, q} \text { does not sep. } N
\end{array}} \kappa_{(\mathcal{U}, \sigma)}\left(a_{1}, \ldots, a_{p+q}\right),
\end{aligned}
$$


where in the last sum not separating $N$ it means that no cycle of $\sigma^{-1} \gamma_{p, q}$ meets both $N_{1}$ and $N_{2}$ but some cycle of $\sigma^{-1} \gamma_{p, q}$ contains more than one point of $N$.

Hence

$$
\begin{gathered}
\sum_{(\mathcal{V}, \pi) \in \mathcal{P} \mathcal{S}_{N C}(p, q)^{\prime}} \kappa_{(\mathcal{V}, \pi)}\left(a_{1}, \ldots, a_{p+q}\right)-\sum_{(\mathcal{V}, \pi) \in \mathcal{P} \mathcal{S}_{N C}(r, s)^{\prime \prime}} \kappa_{(\mathcal{V}, \pi)}\left(A_{1}, \ldots, A_{r+s}\right) \\
=\sum_{\substack{(\mathcal{U}, \sigma) \in \mathcal{P} \mathcal{S}_{N C}(p, q)^{\prime} \\
\sigma^{-1} \gamma_{p, q} \operatorname{sep} . ' s ~}} \kappa_{(\mathcal{U}, \sigma)}\left(a_{1}, \ldots, a_{p+q}\right) .
\end{gathered}
$$

This proves equation (9).

\section{First EXAMPle - THE SQUARE OF A SEMI-CIRCUlar OPERATOR}

Let $(\mathcal{A}, \phi)$ be a unital ${ }^{*}$-algebra with $\phi: \mathcal{A} \rightarrow \mathbb{C}$ a state. A self-adjoint element $x \in \mathcal{A}$ is called semi-circular if $\phi\left(x^{2 k-1}\right)=0$ and $\phi\left(x^{2 k}\right)=\frac{1}{k+1}\left(\begin{array}{c}2 k \\ k\end{array}\right)$ for $k=$ $1,2,3, \ldots$ The element $x$ is called semi-circular because the density of its spectral measure with respect to $\phi$ is $\frac{1}{2 \pi} \sqrt{4-x^{2}}$. This density was shown by E. Wigner to be the limiting eigenvalue distribution of various ensembles of random matrices, in particular of the ensemble usually known as the Gaussian Unitary Ensemble (GUE). This ensemble can be described as follows.

For each positive integer $N$ let $X_{N}=\left(f_{i, j}\right)$ be the complex $N \times N$ self-adjoint random matrix such that

○ $f_{i, j}=x_{i j}+\sqrt{-1} y_{i j}$;

$\circ$ for $i \neq j, x_{i j}$ and $y_{i j}$ are real Gaussian random variables with mean 0 and variance $1 /(2 N)$;

- for each $i, x_{i i}$ is a real Gaussian random variable with mean 0 and variance $1 / N$

○ $\left\{x_{i j}\right\}_{i \leq j} \cup\left\{y_{i j}\right\}_{i<j}$ is a set of independent random variables.

Other descriptions exist; see for example Deift [7, §5.2] or Hiai and Petz [11, §4.1].

The theorem of Wigner asserts that the limiting eigenvalue distribution of $X_{N}$ has the semi-circular density given above. In particular the limiting moments $\left(\alpha_{k}\right)$ are those of a semi-circular operator: $\alpha_{2 k-1}=0$ and $\alpha_{2 k}=\frac{1}{k+1}\left(\begin{array}{c}2 k \\ k\end{array}\right)$ for $k \geq 1$.

In 12 Johansson considered the asymptotic behaviour of the random variables $\left\{\operatorname{Tr}\left(X_{N}^{k}-\alpha_{k} I_{N}\right)\right\}_{k}$ for a class of ensembles containing the GUE. He showed that the random variables were asymptotically Gaussian and showed that the Chebyshev polynomials of the first kind diagonalized the covariance.

In 20] Mingo and Nica showed that the limiting covariances

$$
\alpha_{p, q}=\lim _{N} \mathrm{E}\left(\operatorname{Tr}\left(X_{N}^{p}-\alpha_{p} I_{N}\right) \operatorname{Tr}\left(X_{N}^{q}-\alpha_{q} I_{N}\right)\right),
$$

sometimes called the fluctuation moments, were positive integers which count the number of non-crossing pairings of a $(p, q)$-annulus. These are the elements of $S_{N C}(p, q)$ for which all cycles are of length 2 . These diagrams or equivalent formulations had already been used in a variety of earlier papers: Tutte [26] and [27, 
Jones [13], and King [15, where it was found that

$$
\begin{aligned}
\alpha_{p, q} & =\sum_{k \geq 1} k\left(\begin{array}{c}
p \\
\frac{p-k}{2}
\end{array}\right)\left(\begin{array}{c}
q \\
\frac{q-k}{2}
\end{array}\right) \\
& = \begin{cases}\frac{p q}{2 p+2 q}\left(\begin{array}{c}
p \\
\frac{p}{2}
\end{array}\right)\left(\begin{array}{c}
q \\
\frac{q}{2}
\end{array}\right) & \text { if } p \text { and } q \text { are even, } \\
\frac{(p+1)(q+1)}{8 p+8 q}\left(\begin{array}{c}
p+1 \\
\frac{p+1}{2}
\end{array}\right)\left(\begin{array}{c}
q+1 \\
\frac{q+1}{2}
\end{array}\right) & \text { if } p \text { and } q \text { are odd. }\end{cases}
\end{aligned}
$$

The first expression for $\alpha_{p, q}$ can be seen by observing that every non-crossing pairing of a $(p, q)$-annulus with $k$ through strings (i.e. strings that connect the two circles) can be obtained by connecting a non-crossing pairing of $[p]$ with one block of size $k$ and the others of size 2, with a non-crossing partition of $[q]$ with one block of size $k$ and the others of size 2, and then invoking Kreweras [17, Théorème 2] and finally summing over $k$.

It is natural then to define $\left(\alpha_{p, q}\right)_{p, q}$ to be the fluctuation moments of the semicircular operator.

Let $\mathcal{A}=\mathbb{C}[x]$ be the polynomials in $x$. Define $\phi: \mathcal{A} \rightarrow \mathbb{C}$ by $\phi\left(x^{k}\right)=\alpha_{k}$, where $\left(\alpha_{k}\right)_{k}$ are the moments of the semi-circular operator. Define $\phi_{2}\left(x^{p}, x^{q}\right)=0$ if $p=0$, $q=0$, or $p+q$ is odd and $\phi_{2}\left(x^{p}, x^{q}\right)=\alpha_{p, q}$ (as defined in equation (11)) if $p+q$ is even. One then extends $\phi$ and $\phi_{2}$ by linearity. Notice that the choice of $\phi_{2}$ is independent of $\phi$.

Now $\left(\mathcal{A}, \phi, \phi_{2}\right)$ is a second order non-commutative probability space, and we can consider the cumulants of $x$. It is a standard calculation to see that $\kappa_{n}(x, x, \ldots, x)$ is 0 for all $n \neq 2$ and $\kappa_{2}(x, x)=1$. In the case of second order cumulants, $\left(\kappa_{p, q}\right)_{p, q}$ are all 0 . We can see this directly from equation (8) which states:

$$
\begin{aligned}
\phi_{2}\left(x^{p}, x^{q}\right) & =\sum_{\pi \in S_{N C}(p, q)} \kappa_{\pi}(x, \ldots, x) \\
& +\sum_{(\mathcal{V}, \pi) \in \mathcal{P} \mathcal{S}_{N C}(p, q)^{\prime}} \kappa_{(\mathcal{V}, \pi)}(x, \ldots, x) .
\end{aligned}
$$

Because $x$ is semi-circular, $\kappa_{\pi}(x, \ldots, x)$ is 0 unless $\pi$ is a pairing in which case $\kappa_{\pi}(x, \ldots, x)$ is 1 . Thus the first term on the right hand side of (12) is $\alpha_{p, q}$. Since by definition the left hand side is also $\alpha_{p, q}$ we have that

$$
\sum_{(\mathcal{V}, \pi) \in \mathcal{P S}_{N C}(p, q)^{\prime}} \kappa_{(\mathcal{V}, \pi)}(x, \ldots, x)=0
$$

for all $p$ and $q$. Hence for $p=q=1, \kappa_{1,1}(x, \ldots, x)=0$ as there is only one term. Now $\kappa_{p, q}(x, \ldots, x)$ only appears once in $\sum_{(\mathcal{V}, \pi) \in \mathcal{P} \mathcal{S}_{N C}(p, q)^{\prime}} \kappa_{(\mathcal{V}, \pi)}(x, \ldots, x)$; all the other terms have a factor of $\kappa_{r, s}(x, \ldots, x)$ for either $r<p$ and $s \leq q$ or $r \leq p$ and $s<q$. Thus by induction $\kappa_{p, q}(x, \ldots, x)=0$ for all $p$ and $q$.

Now let $a=x^{2}$. We shall use Theorem 3 to show that the second order cumulants $\kappa_{p, q}=\kappa_{p, q}(a, \ldots, a)$ have the generating function

$$
C(z, w)=\sum_{p, q \geq 1} \kappa_{p, q} z^{p} w^{q}=\frac{z w}{(1-z-w)^{2}},
$$




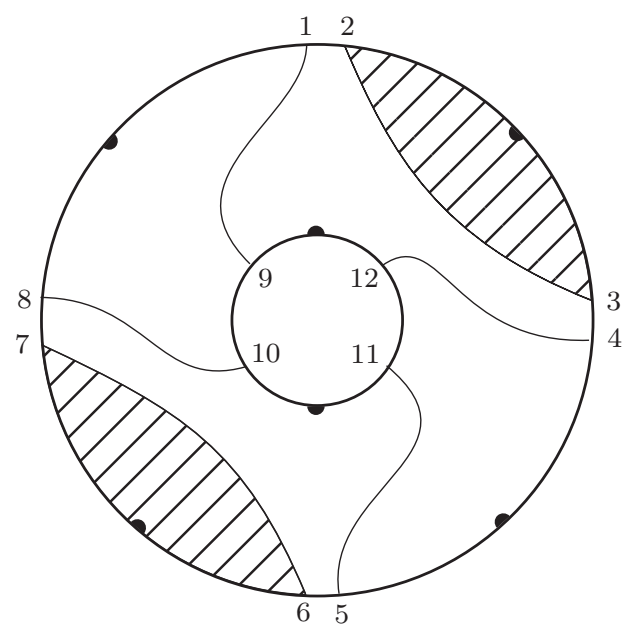

Figure 13. An example of a pairing $\pi$ of the $(8,4)$-annulus such that $\pi^{-1} \gamma_{8,4}$ separates the points of $\{2,4,6,8,10\}$. To construct such a pairing we choose $p-k$ dots on one circle and $q-k$ dots on the other circle and cover these with a pairing (dots 2 and 6 covered with the shading above). All remaining points are connected with through strings. In this example there are only two ways to make the connections: 1 is connected to either 9 or 11 ; this choice forces all the others.

and thus

$$
\kappa_{p, q}=p\left(\begin{array}{c}
p+q-1 \\
p
\end{array}\right) .
$$

First let us recall the first order cumulants of $a$. The square of a semi-circular operator is a special case of a free Poisson operator (with rate 1 in this case). See, for example, Nica-Speicher [23, Propositions 12.11 and 12.13]. As an illustrative example let us show how to use our condition on $\pi^{-1} \gamma_{n}$ to find the first order cumulants of $a$. Let $N=\{2,4,6, \ldots, 2 n\}$. By equation (4) and Theorem 15

$$
\kappa_{n}(a, \ldots, a)=\kappa_{n}\left(x^{2}, \ldots, x^{2}\right)=\sum_{\substack{\pi \in N C(2 n) \\ \pi^{-1} \gamma_{2} \text { sep } N}} \kappa_{\pi}(x, \ldots, x) .
$$

Now as noted above $\kappa_{\pi}(x, \ldots, x)=0$ unless $\pi$ is a pairing. Let $\pi$ be a pairing such that $\pi^{-1} \gamma_{2 n}$ separates the points of $N$. Since $\pi$ is non-crossing 2 is connected to $2 k+1$ for some $k$. Then 2 and $2 k$ are in the same cycle of $\pi^{-1} \gamma_{2 n}$ unless $k=1$, i.e. 2 is connected to 3 . In general we must have that $2 k$ is connected to $2 k+1$ for $1 \leq k<n$ and $2 n$ is connected to 1 . Thus there is only one pairing such that $\pi^{-1} \gamma_{2 n}$ separates the points of $N$. The contribution of this pairing is 1 . Hence $\kappa_{n}(a, \ldots, a)=1$ for all $n$. 
Now let us find the second order cumulants of $a$. Fix $p$ and $q$ and let $N=$ $\{2,4,6, \ldots, 2 p+2 q\}$ :

$$
\begin{aligned}
& \kappa_{p, q}(a, \ldots, a)=\kappa_{p, q}\left(x^{2}, \ldots, x^{2}\right) \\
& =\sum_{\begin{array}{c}
\pi \in S_{N C}(2 p, 2 q) \\
\pi^{-1} \gamma_{2 p, 2 q} \text { sep.'s } N
\end{array}} \kappa_{\pi}(x, \ldots, x)+\sum_{\substack{(\mathcal{V}, \pi) \in \mathcal{P} \mathcal{S}_{N C}(2 p, 2 q)^{\prime} \\
\pi^{-1} \gamma_{2 p, 2 q} \text { sep.'s } N}} \kappa_{(\mathcal{V}, \pi)}(x, \ldots, x) \\
& =\sum_{\substack{\pi \in S_{N C}(2 p, 2 q) \\
\pi^{-1} \gamma_{2 p, 2 q} \text { sep.'s } N}} \kappa_{\pi}(x, \ldots, x)
\end{aligned}
$$

because, as we have shown, all the second order cumulants of $x$ are 0 . Moreover as noted above $\kappa_{\pi}(x, \ldots, x)=0$, except for $\pi$ a pairing in which case we get 1 . Thus $\kappa_{p, q}(a, \ldots, a)$ is the cardinality of $\left\{\pi \in S_{N C}(2 p, 2 q) \mid \pi\right.$ is a pairing and $\pi^{-1} \gamma_{2 p, 2 q}$ separates the points of $N\}$.

Proposition 30. $\kappa_{p, q}(a, \ldots, a)=\sum_{k \geq 1} k\left(\begin{array}{l}p \\ k\end{array}\right)\left(\begin{array}{l}q \\ k\end{array}\right)$.

Proof. We shall show that the number of non-crossing pairings $\pi$ of a $(2 p, 2 q)$ annulus with $k$ pairs of through strings such that $\pi^{-1} \gamma_{2 p, 2 q}$ separates the points of $N=\{2,4,6, \ldots, 2 p+2 q\}$ is $k\left(\begin{array}{l}p \\ k\end{array}\right)\left(\begin{array}{l}q \\ k\end{array}\right)$. Summing over $k$ establishes our claim.

Let $\pi$ be a non-crossing pairing $\pi$ of a $(2 p, 2 q)$-annulus with $k$ pairs of through strings such that $\pi^{-1} \gamma_{2 p, 2 q}$ separates the points of $N$. By the same argument as in the disc case the only possible pairs of $\pi$ which are not through strings are of the form $(2 t, 2 t+1)$. There are $\left(\begin{array}{l}p \\ k\end{array}\right)=\left(\begin{array}{c}p \\ p-k\end{array}\right)$ ways to place these $p-k$ pairs of adjacent elements on $[2 p]$ and $\left(\begin{array}{l}q \\ k\end{array}\right)=\left(\begin{array}{c}q \\ q-k\end{array}\right)$ ways of placing these $q-k$ pairs of adjacent elements on $[2 p+1,2 p+2 q]$. In principle there are $2 k$ ways of connecting the $2 k$ through strings, but half of these put two elements of $N$ in the same cycle of $\pi^{-1} \gamma_{2 p, 2 q}$, so in practice there are only $k$ ways of connecting the through strings. Thus the number of pairings is indeed $k\left(\begin{array}{l}p \\ k\end{array}\right)\left(\begin{array}{l}q \\ k\end{array}\right)$. See Figure 13 .

\section{Theorem 31.}

$$
\sum_{p, q \geq 1} \kappa_{p, q}(a, \ldots, a) z^{p} w^{q}=\frac{z w}{(1-z-w)^{2}}=\sum_{p, q \geq 1} p\left(\begin{array}{c}
p+q-1 \\
p
\end{array}\right) z^{p} w^{q}
$$

Proof.

$$
\begin{aligned}
& \sum_{p, q \geq 1} \kappa_{p, q}(a, \ldots, a) z^{p} w^{q}=\sum_{p, q \geq 1} \sum_{k \geq 1} k\left(\begin{array}{l}
p \\
k
\end{array}\right)\left(\begin{array}{l}
q \\
k
\end{array}\right) z^{p} w^{q} \\
& =\sum_{k \geq 1} k \sum_{p \geq 1}\left(\begin{array}{l}
p \\
k
\end{array}\right) z^{p} \sum_{q \geq 1}\left(\begin{array}{l}
q \\
k
\end{array}\right) w^{q}=\sum_{k \geq 1} k \frac{z^{k}}{(1-z)^{k+1}} \frac{w^{k}}{(1-w)^{k+1}} \\
& =\frac{z w}{[(1-z)(1-w)]^{2}} \sum_{k \geq 1} k\left(\frac{z w}{(1-z)(1-w)}\right)^{k-1} \\
& =\frac{z w}{[(1-z)(1-w)]^{2}} \frac{1}{\left(1-\frac{z w}{(1-z)(1-w)}\right)^{2}}=\frac{z w}{(1-z-w)^{2}} .
\end{aligned}
$$


Continuing we have

$$
\begin{aligned}
\frac{z w}{(1-z-w)^{2}} & =\sum_{k \geq 1} k(z+w)^{k-1} z w=\sum_{k \geq 1} \sum_{p=0}^{k-1} k\left(\begin{array}{c}
k-1 \\
p
\end{array}\right) z^{p+1} w^{k-p} \\
& =\sum_{k, p \geq 1} k\left(\begin{array}{c}
k-1 \\
p-1
\end{array}\right) z^{p} w^{k+1-p} \\
& =\sum_{p, q \geq 1}(p+q-1)\left(\begin{array}{c}
p+q-2 \\
p-1
\end{array}\right) z^{p} w^{q}\left\{\begin{array}{c}
\text { letting } q= \\
k+1-p
\end{array}\right. \\
& =\sum_{p, q \geq 1} p\left(\begin{array}{c}
p+q-1 \\
p
\end{array}\right) z^{p} w^{q} .
\end{aligned}
$$

Let us next outline a method for establishing the same result that uses the second order $R$-transform in equation (1) or the equivalent formulation given in 6 , Equation (52)]. Let $C(z, w)=1+z w R(z, w)$, let $M(z)=\sum_{k>0} \phi\left(a^{k}\right) z^{k}$ be the moment generating function of $a$, and let $M(z, w)=\sum_{p, q \geq 1} \phi_{2}\left(a^{p}, a^{q}\right) z^{p} w^{q}$ be the fluctuation moment generating function of $a$. Then equation (10) becomes

$$
\begin{aligned}
C(z M(z), w M(w))= & \left(M(z, w)+\frac{z w}{(z-w)^{2}}\right) \frac{M(z)}{\frac{d}{d z}(z M(z))} \frac{M(w)}{\frac{d}{d w}(w M(w))} \\
& -\frac{z M(z) w M(w)}{(z M(z)-w M(w))^{2}} .
\end{aligned}
$$

We must find the fluctuation moment generating function $F(z, w)$ of the semicircular operator. Let

$$
F(z)=\sum_{k \geq 0} \phi\left(x^{k}\right) z^{k}=\frac{1-\sqrt{1-4 z^{2}}}{2 z^{2}}
$$

and

$$
\begin{aligned}
F(z, w) & =\sum_{p, q \geq 1} \phi_{2}\left(x^{p}, x^{q}\right) z^{p} w^{q}=\frac{z \frac{d}{d z}(z F(z)) w \frac{d}{d z}(w F(w))}{(1-z F(z) w F(w))^{2}} \\
& =\frac{z w(z F(z)-w F(w))^{2}}{(z-w)^{2}\left(1-z^{2} F(z)^{2}\right)\left(1-w^{2} F(w)^{2}\right)} .
\end{aligned}
$$

Here we obtain the second last expression for $F(z, w)$ by using the first expression for $\alpha_{p, q}$ in equation (11), then Lambert's identity [10, equation 5.21], and finally summing over $k$, and the last expression is obtained from the quadratic equation satisfied by $F$.

Now

$$
M\left(z^{2}\right)=F(z) \text { and } M\left(z^{2}, w^{2}\right)=\frac{1}{2}(F(z, w)+F(-z, w)) .
$$

Thus

$$
M(z, w)=\frac{2 z w M(z)^{3} M(w)^{3}}{(2-M(z))(2-M(w))\left(1-z M(z)^{2} w M(w)^{2}\right)^{2}} .
$$


If we make the substitution $u=z M(z)$ and $v=w M(w)$, then we have

$$
\begin{gathered}
M(z)=\frac{1}{1-u}, z=u-u^{2}, \frac{d}{d z}(z M(z))=\frac{1}{1-2 u}, \text { and } \\
M(z, w)=\frac{2 u v(1-u)(1-v)}{(1-2 u)(1-2 v)(1-u-v)^{2}} .
\end{gathered}
$$

Thus

$$
M(z, w)+\frac{z w}{(z-w)^{2}}=\frac{u v(1-u)(1-v)\left(1-2 u-2 v+2 u^{2}+2 v^{2}\right)}{(1-2 u)(1-2 v)(u-v)^{2}(1-u-v)^{2}} .
$$

After some routine manipulations it follows from (13) and the equation above that

$$
C(u, v)=\frac{u v}{(1-u-v)^{2}} \text {. }
$$

\section{Second EXAmple - A HaAR Unitary}

Let $(\mathcal{A}, \phi)$ be a non-commutative probability space and $u \in \mathcal{A}$ a unitary. Recall (see e.g. [23, Lecture 10]) that $u$ is a Haar unitary if $\phi\left(u^{k}\right)=0$ for $k \neq 0$. In [23] it is shown that for $\epsilon_{1}, \epsilon_{2}, \ldots, \epsilon_{n} \in\{-1,1\}$ then $\kappa_{n}\left(u^{\epsilon_{1}}, u^{\epsilon_{2}}, \ldots, u^{\epsilon_{n}}\right)=0$ unless $n$ is even and $\epsilon_{1}+\epsilon_{2}=\epsilon_{2}+\epsilon_{3}=\cdots=\epsilon_{n-1}+\epsilon_{n}=0$; i.e. all the free cumulants of $\left\{u, u^{*}\right\}$ are 0 except for the alternating ones: $\kappa_{2 n}\left(u, u^{*}, \ldots, u, u^{*}\right)=\kappa_{2 n}\left(u^{*}, u, \ldots, u^{*}, u\right)$ which equal $\mu\left(0_{n}, 1_{n}\right)=(-1)^{n-1} c_{n-1}$, where $\mu$ is the Möbius function of the lattice $N C(n)$ and $c_{n}$ is the $n^{\text {th }}$ Catalan number. Here we wish to indicate the corresponding result for the second order cumulants of $u$ and $u^{*}$. The proofs will be given in a another paper.

We first have to decide how to define the fluctuation moments of a Haar unitary. In [9, Theorem 2], Diaconis and Shahshahani showed that if $U_{N}$ is an $N \times N$ Haar distributed random unitary, then

$$
\mathrm{E}\left(\operatorname{Tr}\left(U_{N}^{k}\right) \operatorname{Tr}\left(U_{N}^{-k}\right)\right)=|k| \text { for } N \geq 2 .
$$

We shall use these fluctuation moments to define our second order Haar unitary.

Definition 32. Let $\left(\mathcal{A}, \phi, \phi_{2}\right)$ be a second order probability space and $u \in \mathcal{A}$ a unitary. We say that $u$ is a second order Haar unitary if $\phi\left(u^{k}\right)=0$ for $k \neq 0$ (i.e. $u$ is a Haar unitary) and for all integers $k, \phi_{2}\left(u^{k}, u^{l}\right)=\delta_{k,-l}|k|$.

Proposition 33. Let $p$ and $q$ be positive integers and $u$ a second order Haar unitary. Let $\epsilon_{1}, \epsilon_{2}, \epsilon_{3}, \ldots, \epsilon_{p+q} \in\{-1,1\}$. Then $\kappa_{p, q}\left(u^{\epsilon_{1}}, u^{\epsilon_{2}}, \ldots, u^{\epsilon_{n-1}}, u^{\epsilon_{n}}\right)=0$ unless $p$ and $q$ are even and

$$
\epsilon_{1}+\epsilon_{2}=\cdots=\epsilon_{p-1}+\epsilon_{p}=\epsilon_{p+1}+\epsilon_{p+2}=\cdots=\epsilon_{p+q-1}+\epsilon_{p+q}=0,
$$

i.e. the $\epsilon$ 's alternate in sign except possibly between $p$ and $p+1$.

As noted above, in the first order case the alternating cumulants of $u$ and $u^{*}$ were given by the Möbius function $\mu$ of the lattice $N C(n)$, i.e.

$$
\kappa_{2 n}\left(u, u^{*}, \ldots, u, u^{*}\right)=\kappa_{2 n}\left(u^{*}, u, \ldots, u^{*}, u\right)=\mu\left(0_{n}, 1_{n}\right) .
$$

We shall state an analogous result for the second order case. Recall from [6, $\S 5.4]$ that the second order Möbius function is defined as the multiplicative function which is the convolution inverse of the zeta function on the set of partitioned permutations. It was shown [6. Theorem 5.24] that $\mu\left(1_{n}, \gamma_{n}\right)=(-1)^{n-1} c_{n-1}$ and $\mu\left(1_{p+q}, \gamma_{p, q}\right)=(-1)^{p+q} c_{p, q}$, where $c_{p, q}$ is the cardinality of $S_{N C}(p, q)$. Moreover it 
was shown [6, page 46] that the the Möbius function satisfies the following recurrence relation:

$$
\begin{aligned}
0= & \mu\left(1_{p+q}, \gamma_{p, q}\right)+q \mu\left(1_{p+q}, \gamma_{p+q}\right) \\
& +\sum_{1 \leq k<p}\left(\mu\left(1_{k+q}, \gamma_{k, q}\right) \mu\left(1_{p-k}, \gamma_{p-k}\right)+\mu\left(1_{k}, \gamma_{k}\right) \mu\left(1_{p-k+q}, \gamma_{p-k, q}\right)\right) .
\end{aligned}
$$

The alternating cumulants of $u$ and $u^{*}$ satisfy the same recurrence relation.

Theorem 34. Let $p=2 m$ and $q=2 n$ be even integers and $\epsilon_{1}, \ldots, \epsilon_{p+q} \in\{-1,1\}$ satisfy (14). Then $\kappa_{p, q}\left(u^{\epsilon_{1}}, \ldots, u^{\epsilon_{p+q}}\right)=(-1)^{m+n} c_{m, n}$.

\section{Concluding Remarks - A Partial order on $\mathcal{P} \mathcal{S}_{N C}(p, q)$}

In $\S 2$ we proved two lemmas (19) and 20) that showed that if $\pi$ is a sub-partition of the Kreweras complement $\sigma^{-1} \gamma_{p, q}$ of $\sigma$, then $\sigma$ is a sub-partition of the Kreweras complement $\gamma_{p, q} \pi^{-1}$ of $\pi$, provided we take the complement on the other side. In Lemma 20 this order was expressed in terms of multiplication of partitioned permutations. The multiplication of partitioned permutations can be used to make $\mathcal{P} \mathcal{S}_{N C}(p, q)$ into a partially ordered set. This order is an extension of the one given in Definition 17.

Definition 35. If $(\mathcal{V}, \pi),(\mathcal{U}, \sigma) \in \mathcal{P} \mathcal{S}_{N C}(p, q)$ we say that $(\mathcal{V}, \pi) \leq(\mathcal{U}, \sigma)$ if there is $\mathcal{W}$ such that $(\mathcal{V}, \pi) \cdot\left(\mathcal{W}, \pi^{-1} \sigma\right)=(\mathcal{U}, \sigma)$.

Proposition 36. Let $(\mathcal{V}, \pi),(\mathcal{U}, \sigma) \in \mathcal{P S}_{N C}(p, q)$ and suppose there is $\mathcal{W}$ such that $(\mathcal{V}, \pi) \cdot\left(\mathcal{W}, \pi^{-1} \sigma\right)=(\mathcal{U}, \sigma)$. Then

(i) $\mathcal{W}=0_{\pi^{-1} \sigma}$ and

(ii) $\mathcal{U}=\mathcal{V} \vee \pi^{-1} \sigma=\mathcal{V} \vee \pi \vee \sigma=\mathcal{V} \vee \sigma \pi^{-1}$

(iii) $\left(0_{\sigma \pi^{-1}}, \sigma \pi^{-1}\right) \cdot(\mathcal{V}, \pi)=(\mathcal{U}, \sigma)$.

Proof. Recall that for $(\mathcal{V}, \pi) \in \mathcal{P S}_{N C}(p, q)$ either $\pi \in S_{N C}(p, q)$ and $\mathcal{V}=0_{\pi}$ or $\pi \in N C(p) \times N C(q)$, and all the blocks of $\mathcal{V}$ contain just one cycle of $\pi$ except one block of $\mathcal{V}$ which contains two cycles of $\pi$ - one from each circle; thus in this case $|\mathcal{V}|=|\pi|+1$. Hence in the former case $|(\mathcal{V}, \pi)|=|\pi|$ and in the latter case $|(\mathcal{V}, \pi)=| \pi \mid+2$.

Thus $|(\mathcal{V}, \pi)|=|\pi|+\delta$, where $\delta=0$ or 2 . By assumption $|(\mathcal{V}, \pi)|+\left|\left(\mathcal{W}, \pi^{-1} \sigma\right)\right|=$ $|(\mathcal{U}, \sigma)|$. If $\sigma \in S_{N C}(p, q)$, then $\mathcal{U}=0_{\sigma}$ and $|(\mathcal{U}, \sigma)|=|\sigma|$. Thus

$$
|\pi|+\delta+\left|\left(\mathcal{W}, \pi^{-1} \sigma\right)\right|=|\sigma| \leq|\pi|+\left|\pi^{-1} \sigma\right|
$$

Therefore $\delta=0$ and $\left|\left(\mathcal{W}, \pi^{-1} \sigma\right)\right|=\left|\pi^{-1} \sigma\right|$, and so $\mathcal{W}=0_{\pi^{-1} \sigma}$.

Suppose that $\sigma \in N C(p) \times N C(q)$; then $|\mathcal{U}|=|\sigma|+1$ and $|(\mathcal{U}, \sigma)|=|\sigma|+2$. If $\pi \in N C(p) \times N C(q)$, then $|(\mathcal{V}, \pi)|=|\pi|+2$, and again we can use the triangle inequality

$$
|\pi|+2+\left|\left(\mathcal{W}, \pi^{-1} \sigma\right)\right|=|\sigma|+2 \leq|\pi|+2+\left|\pi^{-1} \sigma\right|
$$

to conclude that $\mathcal{W}=0_{\pi^{-1} \sigma}$. When $\pi \in S_{N C}(p, q)$ we have to appeal to 6 , Proposition 5.11], and for this we need a little preparation.

Let the cycles of $\sigma$ be $c_{1}, c_{2}, \ldots, c_{k+1}$ and the blocks of $\mathcal{U}$ be $\left\{U_{1}, \ldots, U_{k}\right\}$ with $U_{i}=c_{i}$ as sets for $1 \leq i<k$ and $U_{k}=c_{k} \cup c_{k+1}$ also as sets. Then we can write $\pi=\pi_{1} \pi_{2} \cdots \pi_{k}$ when $\pi$ is the product of the cycles of $\pi$ contained in $U_{i}$. We can 
also decompose $\mathcal{V}$ and $\mathcal{W}$ into $\mathcal{V}_{1}, \mathcal{V}_{2}, \ldots, \mathcal{V}_{k}$ and $\mathcal{W}$ into $\mathcal{W}_{1}, \mathcal{W}_{2}, \ldots, \mathcal{W}_{k}$ along the blocks of $\mathcal{U}$. By the triangle inequality we have that

$$
\left\{\begin{array}{l}
\left|\left(U_{i}, c_{i}\right)\right| \leq\left|\left(\mathcal{V}_{i}, \pi_{i}\right)\right|+\left|\left(\mathcal{W}_{i}, \pi_{i}^{-1} c_{i}\right)\right| \text { for } 1 \leq i<k \text { and } \\
\left|\left(U_{k}, c_{k} c_{k+1}\right)\right| \leq\left|\left(\mathcal{V}_{k}, \pi_{k}\right)\right|+\left|\left(\mathcal{W}_{k}, \pi_{k}^{-1} c_{k} c_{k+1}\right)\right| .
\end{array}\right.
$$

Directly from the definition of $|\cdot|$ we obtain that

$$
\begin{gathered}
|(\mathcal{V}, \pi)|=\left|\left(\mathcal{V}_{1}, \pi_{1}\right)\right|+\cdots+\left|\left(\mathcal{V}_{k}, \pi_{k}\right)\right| \\
|(\mathcal{W}, \sigma)|=\left|\left(\mathcal{W}_{1}, \pi_{1}^{-1} c_{1}\right)\right|+\cdots+\left|\left(\mathcal{W}_{k}, \pi_{k}^{-1} c_{k} c_{k+1}\right)\right|
\end{gathered}
$$

and

$$
|(\mathcal{U}, \sigma)|=\left|\left(\mathcal{U}_{1}, c_{1}\right)\right|+\cdots+\left|\left(\mathcal{U}_{k}, c_{k} c_{k+1}\right)\right| .
$$

Hence

$$
\begin{aligned}
\left|\left(\mathcal{V}_{1}, \pi_{1}\right)\right|+ & \cdots+\left|\left(\mathcal{V}_{k}, \pi_{k}\right)\right|+\left|\left(\mathcal{W}_{1}, \pi_{1}^{-1} c_{1}\right)\right| \\
& +\cdots+\left|\left(\mathcal{W}_{k}, \pi_{k}^{-1} c_{k} c_{k+1}\right)\right|=\left|\left(\mathcal{U}_{1}, c_{1}\right)\right|+\cdots+\left|\left(\mathcal{U}_{k}, c_{k} c_{k+1}\right)\right| .
\end{aligned}
$$

If there were a strict inequality in any of the inequalities (15), then we would have a strict inequality in the equality above. Since we don't, all the inequalities in (15) must be equalities. Hence

$$
\begin{gathered}
\left|\left(U_{i}, c_{i}\right)\right|=\left|\left(\mathcal{V}_{i}, \pi_{i}\right)\right|+\left|\left(\mathcal{W}_{i}, \pi_{i}^{-1} c_{i}\right)\right| \text { for } 1 \leq i<k \text { and } \\
\left|\left(U_{k}, c_{k} c_{k+1}\right)\right|=\left|\left(\mathcal{V}_{k}, \pi_{k}\right)\right|+\left|\left(\mathcal{W}_{k}, \pi_{k}^{-1} c_{k} c_{k+1}\right)\right| .
\end{gathered}
$$

Thus for each $i<k, \mathcal{W}_{i}=0_{\pi_{i}^{-1} c_{i}}$ and $\pi_{i}$ is a non-crossing partition of $c_{i}$. The last equality shows that $\left(\mathcal{V}_{k}, \pi_{k}\right) \cdot\left(\mathcal{W}_{k}, \pi_{k}^{-1} c_{k} c_{k+1}\right)=\left(\mathcal{U}_{k}, c_{k} c_{k+1}\right)$. By [6, Proposition 5.11] we have that $\mathcal{W}_{k}=0_{\pi_{k}^{-1} c_{k} c_{k+1}}$ and thus $\mathcal{W}=0_{\pi^{-1} \sigma}$. Moreover $\left(\mathcal{V}_{k}, \pi_{k}\right)$ is in $\mathcal{P} \mathcal{S}_{N C}\left(c_{k}, c_{k+1}\right)$. This proves $(i)$.

Since $\pi$ and $\sigma$ are permutations, the orbits of the subgroups generated by $\{\pi, \sigma\}$, $\left\{\pi, \pi^{-1} \sigma\right\}$, and $\left\{\pi, \sigma \pi^{-1}\right\}$ are all the same. Thus as partitions $\pi \vee \sigma=\pi \vee \pi^{-1} \sigma=$ $\pi \vee \sigma \pi^{-1}$. Since $\pi \leq \mathcal{V}$ we have $\mathcal{U}=\mathcal{V} \vee \pi^{-1} \sigma=\mathcal{V} \vee \pi \vee \sigma=\mathcal{V} \vee \pi \vee \sigma \pi^{-1}=\mathcal{V} \vee \sigma \pi^{-1}$. This proves that (ii) and (iii) follows from $(i i)$.

Remark 37. Associativity of multiplication of partitioned permutations (6. Proposition 4.10]) shows that the partial order is transitive. Indeed, suppose $(\mathcal{V}, \pi),(\mathcal{U}, \sigma)$, $(\mathcal{W}, \tau) \in \mathcal{P S}_{N C}(p, q)$ and that $(\mathcal{V}, \pi) \leq(\mathcal{U}, \sigma)$ and $(\mathcal{U}, \sigma) \leq(\mathcal{W}, \tau)$. Then

$$
(\mathcal{V}, \pi) \cdot\left(0_{\pi^{-1} \sigma}, \pi^{-1} \sigma\right)=(\mathcal{U}, \sigma) \text { and }(\mathcal{U}, \sigma) \cdot\left(0_{\sigma^{-1} \tau}, \sigma^{-1} \tau\right)=(\mathcal{W}, \tau) .
$$

Then

$$
(\mathcal{V}, \pi) \cdot\left(0_{\pi^{-1} \sigma} \vee 0_{\sigma^{-1} \tau}, \pi^{-1} \tau\right)=(\mathcal{W}, \tau),
$$

and incidentally from Proposition 36 that as partitions $\pi^{-1} \sigma \vee \sigma^{-1} \tau=\pi^{-1} \tau$.

Corollary 38. Let $(\mathcal{V}, \pi),(\mathcal{U}, \sigma) \in \mathcal{P S}_{N C}(p, q)$. Then $(\mathcal{V}, \pi) \leq(\mathcal{U}, \sigma)$ if and only if

(i) $\mathcal{V} \leq \mathcal{U}$;

(ii) if $\sigma \in S_{N C}(p, q)$, then $\pi \in S_{N C}(p, q)$ and every cycle of $\pi$ is contained in a cycle of $\sigma$, and for each cycle of $\sigma$ the enclosed cycles of $\pi$ form a non-crossing permutation of this cycle of $\sigma$; 
(iii) if $\sigma \in N C(p) \times N C(q)$, then every cycle of $\pi$ is contained in either a cycle of $\sigma$ or the union of the two cycles of $\sigma$ connected by $\mathcal{U}$; and for every cycle of $\sigma$ or the union of the two cycles joined by $\mathcal{U}$ the enclosed cycles of $\pi$ form a non-crossing permutation of this cycle or the union of two cycles.

Remark 39. In [6, Notation 5.11] a partitioned permutation $(\mathcal{V}, \pi)$ with $\mathcal{V}=0_{\pi}$ was called a disc permutation and one where $|\mathcal{V}|=|\pi|+1$ was called a tunnel permutation. With our order on $\mathcal{P} \mathcal{S}_{N C}(p, q)$ we see that we can have: $(i)$ disc $\leq$ disc, $(i i)$ disc $\leq$ tunnel; and (iii) tunnel $\leq$ tunnel; but tunnel $\not \leq$ disc.

Remark 40. With this order, $\mathcal{P S}_{N C}(p, q)$ becomes a partially ordered set and the Möbius function of the poset $\mathcal{P} \mathcal{S}_{N C}(p, q)$ has a simple relation to the Möbius function introduced in [6, $\S 5.4]$ and used in $\S 5$. We will address this relation in a forthcoming paper.

\section{REFERENCES}

[1] J. Ambjørn, J. Jurkiewicz, and Yu. M. Makeenko, Multiloop correlators for two-dimensional quantum gravity, Physics Letters B., 251 (1990), 517-524. MR.1084539 (91m:81228)

[2] G. Anderson and O. Zeitouni, A CLT for a band matrix model, Prob. Theory Relat. Fields, 134 (2006), 283-338. MR2222385 (2007j:60031)

[3] Z. D. Bai and J. Silverstein, CLT for linear spectral statistics of large-dimensional sample covariance matrices, Ann. Prob., 32 (2004), 533-605. MR2040792 (2005b:60046)

[4] P. Biane, Some properties of crossings and partitions, Discrete Math., 175 (1997), 41-53. MR:1475837 (98h:05020)

[5] É. Brézin and A. Zee, Universality of the correlations between eigenvalues of large random matrices, Nuclear Phys. B, 402 (1993), 613-627. MR.1236191(95b:82032)

[6] B. Collins, J. A. Mingo, P. Śniady, and R. Speicher, Second order freeness and fluctuations of random matrices: III. Higher order freeness and free cumulants, Documenta Math., 12 (2007), 1-70. MR.2302524

[7] P. Deift, Orthogonal Polynomials and Random Matrices: A Riemann-Hilbert Approach, Courant Lecture Notes in Mathematics, vol. 3, American Mathematical Society, 1998. MR.1677884 (2000g:47048)

[8] P. Diaconis, Patterns in Eigenvalues: The 70th Josiah Willard Gibbs Lecture, Bull. Amer. Math. Soc. (NS), 40 (2003), 155-178. MR.1962294 (2004d:15017)

[9] P. Diaconis and M. Shahshahani, On the eigenvalues of random matrices, J. Appl. Probab., 31A (1994), 49-62. MR1274717 (95m:60011)

[10] R. Graham, D. Knuth, O. Patashnik, Concrete Mathematics, 2nd ed. Addison-Wesley, 1994. MR:1397498 (97d:68003)

[11] F. Hiai and D. Petz, The Semicircle Law, Free Random Variables and Entropy, Mathematical Surveys and Monographs, 77, American Mathematical Society, 2000. MR 1746976 (2001j:46099)

[12] K. Johansson, On fluctuations of eigenvalues of random Hermitian matrices, Duke Math. J., 91 (1998), 151-204. MR1487983 (2000m:82026)

[13] V. F. R. Jones. A quotient of the affine Hecke algebra in the Brauer algebra, Ensiegn. Math., 40 (1994), 313-344. MR1309131 (95j:20038)

[14] A. Khorunzhy, B. Khoruzhenko, and L. Pastur, On the $1 / N$ corrections to the Green functions of random matrices with independent entries, J. Phys. A 28 (1995), L31-L35. MR 1325832 (95m:82075)

[15] C. King. Two-dimensional Potts models and annular partitions, J. Statist. Phy., 96 (1999), 1071-1089. MR1722987(2000m:82009)

[16] B. Krawczyk and R. Speicher, Combinatorics of Free Cumulants, J. Combin. Theory, Ser. A, 90 (2000), 267-292. MR 1757277 (2001f:46101)

[17] G. Kreweras, Sur les partitions non croisées d'un cycle, Discrete Math., 1, 333-350, 1972. MR0309747 (46:8852) 
[18] T. Kusalik, J. A. Mingo, and R. Speicher, Orthogonal polynomials and fluctuations of random matrices, J. Reine Angew. Math., 604 (2007), 1-46. MR2320312

[19] V. P. Leonov and A. N. Shiryaev, On a method of semi-invariants, Theory of Probability and its Applications, 4 (1959), 319-329. MR0123345 (23:A673)

[20] J. Mingo and A. Nica, Annular noncrossing permutations and partitions, and second-order asymptotics for random matrices, Int. Math. Res. Not., 2004 no. 28, 1413-1460. MR2052516 (2005a:46138)

[21] J. Mingo, P. Śniady, and R. Speicher, Second order freeness and fluctuations of random matrices: II. Unitary random matrices, Adv. in Math., 209 (2007), 212-240. MR2294222

[22] J. Mingo and R. Speicher, Second order freeness and fluctuations of random matrices: I. Gaussian and Wishart matrices and cyclic Fock spaces, J. Funct. Anal., 235 (2006), 226-270. MR.2216446 (2007h:46080)

[23] A. Nica and R. Speicher, Lectures on the Combinatorics of Free Probability, London Mathematical Society Lecture Note Series, vol. 335, Cambridge University Press, 2006. MR.2266879

[24] R. Rao, J. Mingo, A. Edelman, and R. Speicher, Statistical eigen-inference from large Wishart matrices, arXiv:math/0701314

[25] R. Speicher, Multiplicative functions on the lattice of noncrossing partitions and free convolution, Math. Ann., 298 (1994), 611-628. MR1268597 (95h:05012)

[26] W.T. Tutte. A census of slicings, Canadian J. Math., 14 (1962), 708-722. MR0142470(26:39)

[27] W.T. Tutte. On the enumeration of planar maps, Bull. Amer. Math. Soc., 74 (1968), 64-74. MR0218276 (36:1363)

[28] D. Voiculescu, Addition of certain non-commuting random variables. J. Funct. Anal., 66 (1986), 323-346. MR839105 (87j:46122)

[29] D. Voiculescu, K. Dykema, A. Nica, Free Random Variables, CRM Monograph Series, Vol. 1, Amer. Math. Soc., 1992. MR1217253(94c:46133)

Department of Mathematics and Statistics, Jeffery Hall, Queen's University, Kingston, Ontario, Canada K7L 3N6

E-mail address: mingo@mast.queensu.ca

Department of Mathematics and Statistics, Jeffery Hall, Queen's University, Kingston, Ontario, Canada K7L 3N6

E-mail address: speicher@mast.queensu.ca

Department of Mathematics and Statistics, Jeffery Hall, Queen's University, Kingston, Ontario, Canada K7L 3N6

E-mail address: 3et8@qlink.queensu.ca 